\title{
Quantum hardness of learning shallow classical circuits
}

\author{
Srinivasan Arunachalam*
}

\author{
Alex B. Grilo ${ }^{+}$
}

\begin{abstract}
In this paper, we study the quantum learnability of constant-depth classical circuits under the uniform distribution and in the distribution-independent framework of PAC learning. In order to attain our results, we establish connections between quantum learning and quantumsecure cryptosystems. We then achieve the following results.
\end{abstract}

1. Hardness of learning $A C^{0}$ and $T C^{0}$ under the uniform distribution. Our first result concerns the concept class $\mathrm{TC}^{0}$ (resp. $\left.\mathrm{AC}^{0}\right)$, the class of constant-depth and polynomialsized circuits with unbounded fan-in majority gates (resp. AND, OR, NOT gates). We show the following:

- if there exists no quantum (quasi-)polynomial-time algorithm to solve the RingLearning with Errors (RLWE) problem, then there exists no (quasi-)polynomial-time quantum learning algorithm for $\mathrm{TC}^{0}$; and

- if there exists no $2^{O\left(d^{1 / \eta}\right)}$-time quantum algorithm to solve RLWE with dimension $d=$ $O($ polylog $n)$ (for every constant $\eta>2)$, then there exists no $O\left(n^{\log ^{v} n}\right)$-time quantum learning algorithm for poly $(n)$-sized $\mathrm{AC}^{0}$ circuits (for a constant $v>0$ ), matching the classical upper bound of Linial et al. [LMN93]

where the learning algorithms are under the uniform distribution (even with access to quantum membership queries). The main technique in these results uses an explicit family of pseudo-random functions that are believed to be quantum-secure to construct concept classes that are hard to learn quantumly under the uniform distribution.

2. Hardness of learning $\mathrm{TC}_{2}^{0}$ in the PAC setting. Our second result shows that if there exists no quantum polynomial-time algorithm for the LWE problem, then there exists no polynomial-time quantum-PAC learning algorithm for the class $\mathrm{TC}_{2}^{0}$, i.e., depth- $2 \mathrm{TC}^{0}$ circuits. The main technique in this result is to establish a connection between the quantum security of public-key cryptosystems and the learnability of a concept class that consists of decryption functions of the cryptosystem.

Our results show that quantum resources do not give an exponential improvement to learning constant-depth polynomial-sized neural networks. This also gives a strong (conditional) negative answer to one of the "Ten Semi-Grand Challenges for Quantum Computing Theory" raised by Aaronson [Aar05].

\footnotetext{
${ }^{*}$ Center for Theoretical Physics, MIT. Funded by the MIT-IBM Watson AI Lab under the project Machine Learning in Hilbert space. arunacha@mit.edu

${ }^{\dagger}$ CWI and QuSoft, Amsterdam, The Netherlands. Supported by ERC Consolidator Grant 615307-QPROGRESS. alexg@cwi.nl

${ }^{\ddagger}$ Joint Center for Quantum Information and Computer Science, University of Maryland, USA. Supported by the Department of Defense. aarthims@umd.edu
} 


\section{Contents}

1 Introduction $\quad 1$

1.1 Learning models . . . . . . . . . . . . . . . . . . . . . 3

1.2 Strengths of quantum examples under the uniform distribution . . . . . . . . . . 4

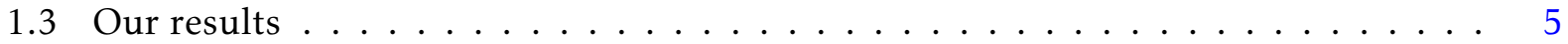

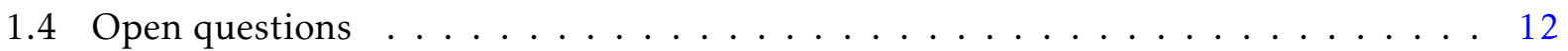

1.5 Differences with previous version $\ldots \ldots \ldots \ldots \ldots \ldots$

2 Preliminaries $\quad 14$

2.1 Notation and basic claims . . . . . . . . . . . . . . . . . . . . . 14

2.2 Information theory and communication complexity . . . . . . . . . . . 14

2.3 Cryptographic primitives . . . . . . . . . . . . . . 15

2.4 Learning models . . . . . . . . . . . . . . . . . . . 16

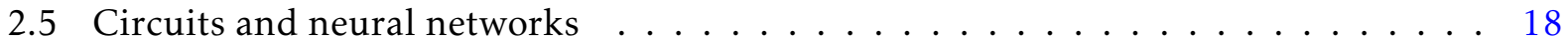

3 Hardness assumptions and cryptographic constructions 20

3.1 Learning with Errors $(\mathrm{LWE}) \ldots \ldots \ldots \ldots \ldots \ldots$

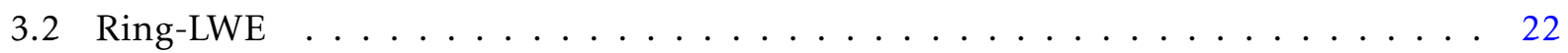

3.3 Learning with Rounding $(\mathrm{LWR}) \ldots \ldots \ldots \ldots \ldots$

4 Quantum-secure PRFs vs. quantum learning 28

4.1 Hardness of learning $\mathrm{TC}^{0} \ldots \ldots \ldots \ldots \ldots \ldots \ldots \ldots \ldots \ldots$

4.2 Hardness of learning $\mathrm{AC}^{0} \ldots \ldots \ldots \ldots \ldots \ldots \ldots \ldots \ldots \ldots$

5 Quantum-secure encryption vs. quantum learning 34

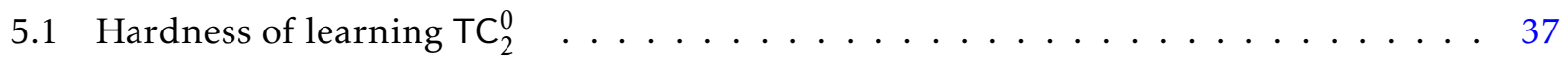

A Time-efficient learning of $\mathrm{NC}^{0} \quad 42$

\section{Introduction}

Machine learning is a diverse field of research with many real-world applications and has received tremendous attention in the past decade. From a theoretical perspective, since the seminal paper of Valiant [Val84], there has been a lot of theoretical effort in considering different learning models that formalize what we mean by learning and understanding which problems can (or cannot) be efficiently learned within these models.

In recent years, due to the considerable development of quantum computation (both on the theoretical and experimental fronts), there has been an increased focus on understanding the tasks for which quantum computers can offer speedups. Machine learning tasks have emerged as a candidate in this respect. To this end, results in quantum learning theory aim to identify learning problems for which quantum computers provably provide a (significant) advantage.

More concretely, in learning theory, the goal is to devise a learning algorithm (or learner) for a 
set of functions which is called a concept class. The functions in the concept class $\mathcal{C}$ are referred to as concepts. In this paper, we will consider (without loss of generality) concepts that are Boolean functions $c:\{0,1\}^{n} \rightarrow\{0,1\}$. The learner is provided with examples of the form $(x, c(x))$, where $c$ is an unknown concept lying in $\mathcal{C}$ and $x$ is picked uniformly at random from $\{0,1\}^{n}$ and the goal of the learner is to learn $c$, i.e., it should output a hypothesis $h$ that is close to $c .{ }^{1}$ We say that a learner $\mathcal{A}$ learns $\mathcal{C}$, if for every $c \in \mathcal{C}, \mathcal{A}$ learns $c$. In learning theory, the intent is to devise efficient learning algorithms for an interesting concept class $\mathcal{C}$, i.e., the learner should use few examples and not too much time in order to learn $\mathcal{C}$.

In quantum learning theory, the goal is still to learn concept classes $\mathcal{C}$, but now with the access to quantum resources. Bshouty and Jackson [BJ99] introduced a quantum learning model wherein the learner is a quantum algorithm and is provided with quantum examples of the form

$$
\frac{1}{\sqrt{2^{n}}} \sum_{x \in\{0,1\}^{n}}|x, c(x)\rangle
$$

for some concept $c \in \mathcal{C}$. In order to see why quantum examples generalize classical examples, observe that a quantum learner could choose to measure the quantum example in the computational basis, which results in a classical example $(x, c(x))$ for a uniformly random $x \in\{0,1\}^{n}$. The advantage of quantum learning usually comes from the fact that one can perform arbitrary unitary operations on these quantum examples, enabling one to improve sample or time complexity for learning the concept class $\mathcal{C}$.

The first example of a quantum advantage for a learning problem was showed by Bernstein and Vazirani [BV97]. They showed how to learn the concept class of linear functions $\mathcal{C}=\left\{c(x)=\sum_{i} s_{i} x_{i}\right.$ $\left.\bmod 2: s \in\{0,1\}^{n}\right\}$ with a constant number of quantum examples. Classically, in order to learn this concept class efficiently, it is necessary and sufficient to obtain $\widetilde{\Theta}(n)$ examples. With these many examples a classical learner can use Gaussian elimination and learn the unknown target concept in polynomial time.

Subsequently, Bshouty and Jackson [BJ99] showed that the class of Boolean functions that can be represented as polynomial-sized DNF formulas can be learned in quantum polynomial time. ${ }^{2}$ A crucial ingredient for their quantum learning algorithm was the ability to perform Fourier sampling using quantum examples (which we discuss in Section 1.2). Classically, Verbeurgt [Ver90] showed that DNFs can be learned in quasi-polynomial time using classical examples and this has remained the state-of-the art for the last thirty years! This emphasizes the power of quantum examples for learning. There have been many other instances (which we discuss in Section 1.2) where quantum examples give an advantage for quantum learning algorithms. A natural followup question to the quantum-efficient learnability of DNF formulas, i.e., depth -2 circuits, is:

Can the concept class of shallow, i.e., constant-depth, classical circuits be learned more efficiently using quantum resources as compared to classical resources?

In particular, are there efficient (i.e., polynomial-time) quantum learning algorithms for all of the Boolean functions that can be represented by $\mathrm{AC}^{0}$ circuits, i.e., constant-depth circuits with unbounded fan-in AND, OR, NOT gates? More ambitiously, can we quantum-efficiently learn $\mathrm{TC}^{0}$, i.e., constant-depth circuits with majority gates. ${ }^{3}$ This question was raised by Aaronson [Aar05] as one of the "Ten Semi-Grand Challenges for Quantum Computing Theory”.

\footnotetext{
${ }^{1}$ More formally, this is referred to as the uniform-distribution learning setting. In learning theory there are several variations of this model that we skip for the sake of simplicity. See Section 1.1 for a brief introduction and Section 2.4 for more details.

${ }^{2} \mathrm{~A}$ DNF formula is a disjunction of conjunctions of variables and their negations.

${ }^{3}$ A majority gate on $n$ bits outputs 1 if $\lfloor n / 2\rfloor+1$ bits evaluate to 1 and 0 otherwise.
} 
We notice that understanding the learnability of $\mathrm{TC}^{0}$, apart from being theoretically important, also sheds light on the question: can quantum computers help learn the weights of neural networks faster? It is well-known [MSS91, GHR92] that constant-depth polynomial-sized feedforward neural networks, where the weights of neurons are bounded by a polynomial in the input size, can be implemented by $\mathrm{TC}^{0}$ circuits.

In this work we address this question and give evidence that efficient quantum algorithms do not exist for learning TC ${ }^{0}$. More concretely, under the assumption that the Ring-Learning with Errors problem (RLWE) [LPR13a] cannot be solved in (quasi-)polynomial time by quantum computers, the class of $\mathrm{TC}^{0}$ functions cannot be weak-learned in quantum (quasi-)polynomial time. Also, under the less-standard assumption that RLWE cannot be solved in strongly sub-exponential time, by quantum computers, we show that there exists a constant $v>0$ such that there is no $n^{O\left(\log ^{v} n\right) \text { - }}$ time quantum learning algorithm for $\mathrm{AC}^{0}$. Here, the "strongly sub-exponential" time hardness for RLWE is defined as follows: for every constant $\eta>2$, the $d$-dimensional RLWE problem cannot be solved in $2^{O\left(d^{1 / \eta}\right)}$-time by quantum computers. RLWE is one of the leading candidates for efficient post-quantum cryptography and it is believed to be hard to solve, even for quantum computers. For instance, the current best-known quantum algorithms for it [Sta19] do not, asymptotically, perform any better than the lattice reduction techniques used for the Learning with Errors problem [CN11, LLL82].

\subsection{Learning models}

In this section, we first recall the definitions of the classical and quantum learning models. For a detailed introduction to these models, we refer the reader to Section 2.4.

Classical learning model. In the Probably Approximately Correct (PAC) model of learning proposed by Valiant [Val84], a concept class $\mathcal{C}$ is a subset of Boolean functions, i.e., $\mathcal{C} \subseteq\left\{c:\{0,1\}^{n} \rightarrow\right.$ $\{0,1\}\}$ and every element $c:\{0,1\}^{n} \rightarrow\{0,1\}$ in $\mathcal{C}$ is referred to as a concept. The goal of the learning algorithm $\mathcal{A}$ is to learn an unknown target concept $c \in \mathcal{C}$ given labeled examples of the form $(x, c(x))$ where $x$ is drawn from an unknown distribution $D:\{0,1\}^{n} \rightarrow[0,1]$. We say that a learning algorithm $\mathcal{A}$ learns $\mathcal{C}$ if it satisfies the following: for every $c \in \mathcal{C}$ and every distribution $D:\{0,1\}^{n} \rightarrow[0,1]$, with probability at least $2 / 3, \mathcal{A}$ outputs a hypothesis $h:\{0,1\}^{n} \rightarrow\{0,1\}$ that satisfies $\operatorname{Pr}_{x \sim D}[h(x)=c(x)] \geq 1-\varepsilon$. The advantage of the learner over a random guess is $\beta:=\frac{1}{2}-\frac{\varepsilon}{2}$ and $\beta$ is called the bias of the learner. The learner $\mathcal{A}$ properly learns the concept class $\mathcal{C}$ if its output hypothesis is always in the concept class, i.e., $h \in \mathcal{C}$. Otherwise, $\mathcal{A}$ is an improper learner for $\mathcal{C}$. Similarly, $\mathcal{A}$ is a weak learner if $\beta=n^{-c}$ for some constant $c>0$ and $\mathcal{A}$ is a strong learner if $\beta \geq 1 / 6$. In this paper all our lower bounds will be for improper learners and, unless explicitly stated, for weak learners.

The sample complexity of a learning algorithm $\mathcal{A}$ is the worst-case number of labeled examples it uses and the time complexity of $\mathcal{A}$ is the worst-case running time (where the worst-case is taken with respect to the hardest concept $c \in \mathcal{C}$ and distribution $D$ ). The sample/time complexity of a concept class $\mathcal{C}$ is the sample/time complexity of the most efficient learning algorithm for $\mathcal{C}$.

In this work, we also consider learning models that relax the PAC learning framework in two ways. First we allow the learner to make membership queries to a target concept $c$, i.e., $\mathcal{A}$ is allowed to ask "what is $c(x)$ " for an arbitrary $x$ of its choice. Second, instead of the learner succeeding under every distribution $D$, we consider the learnability of $\mathcal{C}$ when $D$ is fixed and known to the learner. We say that $\mathcal{A}$ PAC-learns $\mathcal{C}$ under $D$ with membership queries if: for every $c \in \mathcal{C}$, with probability $\geq 2 / 3, \mathcal{A}$, takes labeled examples and makes membership queries, and outputs a hypothesis $h$ such that $\operatorname{Pr}_{x \sim D}[h(x) \neq c(x)] \leq \varepsilon$. 
Quantum learning model. Bshouty and Jackson [BJ99] introduced the model of quantum-PAC learning, which naturally generalizes the classical PAC model. Here, a quantum learning algorithm $\mathcal{A}$ has to learn the unknown concept $c \in \mathcal{C}$ given quantum examples of the form

$$
\sum_{x} \sqrt{D(x)}|x, c(x)\rangle
$$

where $D:\{0,1\}^{n} \rightarrow[0,1]$ is an unknown distribution. The goal of the quantum learner and the notion of sample and time complexities are analogous to the classical model.

As described in the classical setting, we also consider the model where the quantum learning algorithm is given access to an oracle $O_{c}:|x, b\rangle \rightarrow|x, b \oplus c(x)\rangle$ that allows it to make quantum membership queries. Additionally, instead of requiring the learner to succeed for all distributions $D$, we also consider quantum learners that learn $\mathcal{C}$ under a fixed distribution $D$. For quantum learning algorithms, a natural choice of $D$ is the uniform distribution over $\{0,1\}^{n}$, and in this case a quantum example is given by $\frac{1}{\sqrt{2^{n}}} \sum_{x}|x, c(x)\rangle$. We discuss the utility of such examples in the next section. We say a learner $\mathcal{A}$ uniform quantum-PAC learns $\mathcal{C}$ with membership queries if: for every $c \in \mathcal{C}$, with probability $\geq 2 / 3, \mathcal{A}$ uses uniform quantum examples and quantum membership queries to output a hypothesis $h$ such that $\operatorname{Pr}_{x \sim D}[h(x) \neq c(x)] \leq \varepsilon$.

\subsection{Strengths of quantum examples under the uniform distribution}

One of the main tools in quantum learning theory, when considering learning under the uniform distribution, is the ability to efficiently perform Fourier sampling. In order to explain it, we first introduce the following. For a Boolean function $c:\{0,1\}^{n} \rightarrow\{-1,1\}$, the Fourier coefficients of $c$ are given by $\widehat{c}(S)=\frac{1}{2^{n}} \sum_{S} c(x)(-1)^{x \cdot S}$. The Fourier distribution $\left\{\widehat{c}(S)^{2}: S \in\{0,1\}^{n}\right\}$ is given by the squared Fourier coefficients of $c$ and they satisfy $\sum_{S} \widehat{c}(S)^{2}=1$. Fourier sampling refers to the task of sampling from the Fourier distribution $\left\{\widehat{c}(S)^{2}\right\}_{S}$.

An advantage of having uniform quantum examples is that, using standard ideas from quantum information theory, a quantum learner can efficiently perform the operation

$$
\frac{1}{\sqrt{2^{n}}} \sum_{x}|x, c(x)\rangle \rightarrow \sum_{S} \widehat{c}(S)|S\rangle
$$

given $O(1)$ copies of $\frac{1}{\sqrt{2^{n}}} \sum_{x}|x, c(x)\rangle$. Measuring the resulting state allows a quantum learner to obtain a sample from the Fourier distribution. Hence, using uniform quantum examples, one can sample from the Fourier distribution $\left\{\widehat{c}(S)^{2}\right\}_{S}$. Classically, we do not know how to perform this sampling process (or even approximately sample) efficiently, since the Fourier coefficients $\widehat{c}(S)$ depends on $2^{n}$ values of $x$. Therefore, one avenue to obtain quantum speedups with uniform quantum examples arises from the use of Fourier sampling.

Indeed, quantum Fourier sampling has been profitably used in many applications. This idea was first used in the aforementioned paper of Bernstein and Vazirani [BV97], where they observe that the Fourier support of linear functions is concentrated on a single point. Therefore, unknown linear functions can be learned using just one uniform quantum example. Fourier sampling was later used by Bshouty and Jackson [BJ99] to show that DNFs can be learned quantum-efficiently. A classical analogue of this question is a long-standing open question. ${ }^{4}$ Kanade et al. [KRS18] extended the result of Bshouty and Jackson and showed how to learn DNFs quantum-efficiently even under product distributions.

In fact, a notorious bottleneck in classical learning theory is obtaining a polynomial time

\footnotetext{
${ }^{4}$ With classical membership queries, DNF formulas can be learned in classical polynomial time [Jac97].
} 
learning algorithm for the class of $O(\log n)$-juntas ${ }^{5}$ which are a subset of polynomial-sized DNFs. By contrast, Atic1 and Servedio [AS09] showed that $O(\log n)$-juntas can be learned quantumefficiently under the uniform distribution. In fact the time-efficient learnability of $O(\log n)$-juntas can be used to time-efficiently quantum learn the concept class $\mathrm{NC}^{0}$ under the uniform distribution (for a proof of this, see Appendix A). ${ }^{6}$ Subsequently, Arunachalam et al. $\left[\mathrm{ACL}^{+} 19\right]$ showed that the concept class of $k$-Fourier-sparse Boolean functions (which includes both $(\log k)$-juntas and linear functions) can be learned query-efficiently given quantum examples.

One thing common to all these results was the application of the Fourier sampling to learn a concept class under the uniform distribution, which was key for the large quantum speedup.

Related work. In the context of learning, apart from the uniform distribution setting, some works have focused on understanding the power of quantum learning under arbitrary distributions [SG04, AS05, AW18]. In particular, [AW18] showed that quantum examples do not provide an advantage over classical examples for PAC learning.

Recently, both shallow circuits and the Learning with Errors problem have been used in different contexts to understand the capabilities of quantum computation. Grilo et al. [GKZ19] showed that polynomially many quantum examples suffice to solve the LWE problem in quantum polynomial time, while this remains a hard problem when given just classical examples. Bravyi et al. [BGK18] exhibited a problem which can be solved using shallow quantum circuits but requires logarithmic depth classical circuits (with bounded fan-in) to solve. Bene Watts et al. [BKST19] improved their result by removing the "bounded fan-in" assumption. In another context, under the assumption that the Learning with Errors problem is hard for quantum computers (given just classical examples), Mahadev [Mah18] demonstrated a classical protocol to classically verify the result of an efficient quantum computation.

\subsection{Our results}

In this paper we address two natural questions that arise from the work of Bshouty and Jackson [BJ99], which showed the quantum-efficient learnability of depth-2 circuits.

The first question is, can the work of [BJ99] be extended to learn depth-3 circuits, or more generally, constant-depth polynomial-sized circuits (i.e., $\mathrm{TC}^{0}$ and $\mathrm{AC}^{0}$ ) in quantum polynomial time? Classically, in a seminal result, Linial, Mansour and Nisan [LMN93] constructed an $n^{O(\operatorname{polylog} n)}$ time learning algorithm for $\mathrm{AC}^{0}$ by approximately learning the Fourier spectrum of an unknown $\mathrm{AC}^{0}$ circuit. Subsequently, Kharitonov [Kha93] showed that their learning algorithm is optimal assuming that factoring cannot be solved in quasi-polynomial time. Since factoring can be solved in quantum polynomial time with Shor's algorithm [Sho97], the lower bound of Kharitonov doesn't apply to quantum learners. Moreover, since Fourier sampling is easy quantumly (under the uniform distribution), it seems plausible that one could efficiently learn important properties of the Fourier spectrum of $A C^{0}$ circuits (similar to the work of Linial et al. [LMN93]). This could possibly result in efficient quantum learning algorithms for $\mathrm{AC}^{0}$ under the uniform distribution (similar to many results discussed in the previous section).

The second question is, can the work of [BJ99] be extended to the class of depth-2 threshold circuits (known as $\mathrm{TC}_{2}^{0}$ ), i.e., can $\mathrm{TC}_{2}^{0}$ be learned quantum-efficiently? We notice that $\mathrm{TC}^{0}$ circuits are practically very relevant since constant-depth polynomial-sized feed-forward neural networks with weights (of the neurons) bounded by some polynomial in the input size, can be implemented

\footnotetext{
${ }^{5} \mathrm{~A} k$-junta is a Boolean function on $n$ bits, whose output only depends on $k$ out of the $n$ input bits.

${ }^{6} \mathrm{NC}^{0}$ is the concept class of constant-depth circuits consisting of fan-in 2 AND, OR, NOT gates.
} 
as circuits in $\mathrm{TC}^{0}$ [MSS91, GHR92]. If there were efficient quantum algorithms for learning $\mathrm{TC}^{0}$, then it is plausible that quantum computers could give an enormous advantage in approximately learning the weights for neural networks.

In this paper, we give a conditional negative answer to both questions. In particular, we show that under the assumptions that support the security of current post-quantum cryptography: $\mathrm{TC}^{0}$ and $\mathrm{AC}^{0}$ cannot be learned efficiently by quantum computers under the uniform distribution (under different assumptions); and $\mathrm{TC}_{2}^{0}$ cannot be PAC learned efficiently by quantum computers. We summarize these results in the table below. ${ }^{7}$

These results give a strong negative answer to a question of Aaronson [Aar05], under crypto-

\begin{tabular}{|c|c|c|c|}
\hline No learner in this model & Running in & $\begin{array}{c}\text { For the } \\
\text { complexity class }\end{array}$ & Assuming \\
\hline \multirow{3}{*}{$\begin{array}{c}\text { Uniform-distribution } \\
\text { PAC } \\
\text { (with membership queries) }\end{array}$} & $\begin{array}{c}n^{O\left(\log ^{v} n\right)} \text { time for a } \\
\text { constant } v>0\end{array}$ & $A C^{0}$ & $\begin{array}{l}\text { No strongly sub-exponential time } \\
\text { algorithm for RLWE }\end{array}$ \\
\hline & $2^{O(\operatorname{polyl} \log n)}$ time & $\mathrm{TC}^{0}$ & $\begin{array}{l}\text { No quasi-polynomial time } \\
\text { algorithm for RLWE }\end{array}$ \\
\hline & $\operatorname{poly}(n)$ time & $\mathrm{TC}^{0}$ & RLWE $\notin B Q P$ \\
\hline Distribution-free PAC & $\operatorname{poly}(n)$ time & $\mathrm{TC}_{2}^{0}$ & $\mathrm{LWE} \notin \mathrm{BQP}$ \\
\hline
\end{tabular}

Table 1: Hardness of learning results for $\operatorname{poly}(n)$-sized circuits.

graphic assumptions. Aaronson asked if, $\mathrm{TC}^{0}$ and $\mathrm{AC}^{0}$ can be quantum-PAC learned in polynomial time. Our first result already gives a (conditional) negative answer even when we fix the uniform distribution and allow the learner to make quantum membership queries. Our second result gives a conditional refutation to the PAC learnability of $\mathrm{TC}^{0}$ even when restricted to depth- 2 threshold circuits.

In order to achieve our results, we follow a strategy proposed by Valiant [Val84], who showed the hardness of proper learning the class of polynomial-sized circuits based on the security of cryptographic objects. This strategy was subsequently improved upon to give conditional improper learning lower bounds and used to prove the classical hardness of various concept classes [Kha92, Kha93, KV94, KS09]. These results have the following structure: assuming there exists an efficient learning algorithm for some concept class $\mathcal{C}$, there exists an adversary that is able to break some cryptographic construction using the learning algorithm as a subroutine. Here, the adversary provides the resources to the learning algorithm based on the cryptographic primitive it is trying to break. This implies that if the cryptographic construction is secure, then such a learning algorithm cannot exist.

In this paper, we quantize these well-studied classical proof-of-hardness techniques. The difficulties in quantizing such results are three-fold. First, many of the classical hardness of learning results rely on cryptographic primitives whose security is based on the hardness of factoring. As stated previously, this would not hold in the quantum regime due to Shor's quantum polynomialtime factoring algorithm [Sho97]. Second, the fact that adversaries can efficiently create classical examples from some distribution $D$ does not imply that quantum examples can be created according to the same distribution. An important issue we run into in this case is solving the indexerasure problem, which is known to be hard to solve on quantum computers [AMRR11, LR19]. Finally, some of the hardness results implicitly use the fact that the learning algorithm they are considering is classical and the proof techniques do not follow through in the quantum setting.

\footnotetext{
${ }^{7} \mathrm{BQP}$, or bounded-error quantum polynomial time, is the class of decision-problems that be solved in polynomial time on a quantum computer with bounded-error.
} 
For example, Kharitonov [Kha93] uses collision arguments to bound the amount of information retrieved by the learner, but this approach does not work quantumly. We discuss these issues in further detail in the next section.

In subsequent sections, we delineate the connections between the hardness of quantum learning and the security of certain cryptographic primitives - specifically, quantum-secure family of pseudo-random functions and public-key encryption schemes. Next, we sketch how to use these connections to show hardness of quantum learning for some interesting concept classes.

\subsubsection{Pseudo-random functions vs. quantum learning}

A family of pseudo-random functions (PRF) are cryptographic objects that "mimic" random functions. More concretely, a PRF is a keyed-family of functions $\mathcal{F}=\left\{f_{\mathbf{k}}:\{0,1\}^{n} \rightarrow\{0,1\}^{\ell}\right.$ where $\mathbf{k} \in$ $\left.\{0,1\}^{M}\right\}$ such that no efficient adversary that is given query access to $f$ can distinguish if $f$ was uniformly picked from $\mathcal{F}$ or a truly random function with non-negligible advantage over a random guess. ${ }^{8}$ If even polynomial time quantum adversaries cannot distinguish such cases with quantum query access to the function, then we say that the PRF is quantum-secure. We informally state our first result below (see Corollary 4.3 for a full statement).

Result 1 If there is a quantum-secure PRF $\mathcal{F}$, then $\mathcal{F}$ does not have an efficient uniform quantum-PAC learner with membership queries.

Kharitonov [Kha93] established the connection between Pseudo-random generators (PRGs) and classical learning by constructing a circuit class such that the PRG is computed by the circuit class. He proceeded to show that if a learning algorithm for such a concept class existed, then would be possible to break the PRG. This approach (implicitly) requires that while the complexity of the PRG scales polynomially with the size of the seed, it achieves a super-polynomial stretch Such a requirement is satisfied, for instance, by the BBS PRG [BBS86], whose security relies on hardness of factoring.

Unfortunately, no post-quantum PRGs are known with this property. Inspired by the ideas used in [Kha93], we overcome this by considering the connection between quantum-secure PRFs and we prove Result 1. We stress that the proof in [Kha93] does not trivially quantize. For instance, the crux in Kharitonov's proof is determining the probability of collision in the classical examples, and this does not make sense in the quantum regime. Clearly, each quantum example contains information about every input. However, we can show that efficiently accessing this information simultaneously for most $x$ is information-theoretically impossible.

We now sketch a proof of Result 1 . Let $\mathcal{F}=\left\{f_{\mathbf{k}}:\{0,1\}^{n} \rightarrow\{0,1\}\right.$ where $\left.\mathbf{k} \in\{0,1\}^{M}\right\}$. By contradiction, let us assume there exists an efficient uniform-PAC quantum learning algorithm for $\mathcal{F}$. Using this efficient learner, we show how to construct a quantum distinguisher for the PRF $\mathcal{F}$. As $\mathcal{F}$ is assumed to be quantum-secure, we obtain a contradiction and prove the result. More concretely, let $f$ be a a function and suppose a distinguisher with access to $f$ has to determine if it comes from $\mathcal{F}$ or it is a truly random function. Consider a distinguisher that simulates a quantum learning algorithm $\mathcal{A}$ by using his oracle to answer the learner's queries. $\mathcal{A}$ is supposed to output a hypothesis $h$ that approximates $f$. The distinguisher then picks a random challenge $x^{*} \in\{0,1\}^{n}$ and outputs 1 if and only if $h\left(x^{*}\right)=f\left(x^{*}\right)$. One technical aspect that we show here is that, if the learning algorithm has bias $\beta(n)$, then the distinguisher has an advantage $\geq \frac{\beta(n)}{2}$ in distinguishing if $f$ comes from the pseudo-random function family or is a random function. In particular, if

\footnotetext{
${ }^{8}$ By negligible advantage, we mean: for every $c>0$ the advantage is at most $1 / n^{c}$.
} 
there is a polynomial time weak quantum learning algorithm for $\mathcal{F}$ under the uniform distribution, then there exists a polynomial-time quantum distinguisher $\mathcal{D}$ and some constant $c>0$ that satisfies

$$
\left|\operatorname{Pr}_{f \in \mathcal{F}}\left[\mathcal{D}^{|f\rangle}(\cdot)\right]-\operatorname{Pr}_{f \in \mathcal{U}}\left[\mathcal{D}^{|f\rangle}(\cdot)\right]\right| \geq \frac{1}{n^{c}},
$$

where, in the first case, $f$ is picked uniformly at random from $\mathcal{F}$ and, in the second case, $f$ is a uniformly random function. The key point in order to prove that a quantum learner with advantage $\beta(n)$ implies a distinguisher with advantage $\frac{\beta(n)}{2}$ is to bound the success probability of learning a random function.

\subsubsection{Public-key encryption schemes vs. quantum learning}

A public-key encryption scheme consists of a triple of algorithms (Key-generator, Enc, Dec). Keygenerator is a randomized algorithm that on input $1^{n}$ (where $n$ is a security parameter) outputs a tuple $\left(\mathrm{K}_{\text {pub }}, \mathrm{K}_{\text {priv }}\right)$, where $\mathrm{K}_{\text {pub }}$ is a publicly known key used to encrypt messages and $\mathrm{K}_{\text {priv }}$ is a private key used to decrypt messages. Enc is a deterministic algorithm that receives as input the public key $\mathrm{K}_{\text {pub }}$, some randomness $r$ and a message $b \in\{0,1\}$ and outputs Enc $\left(\mathrm{K}_{\text {pub }}, r, b\right)$, which we denote by $\mathrm{Enc}_{\mathrm{K}_{\mathrm{pub}}}(r, b)$ for simplicity. Dec receives as input the private key $\mathrm{K}_{\mathrm{priv}}$ and $\mathrm{Enc}_{\mathrm{K}_{\mathrm{pub}}}\left(r^{*}, b^{*}\right)$ and outputs $c \in\{0,1\}$ (we write $\operatorname{Dec}_{K_{\text {priv }}}$ in order be explicit about the dependence of Dec on $\mathrm{K}_{\text {priv }}$ ). The public-key encryption scheme is said to be correct if: for uniformly random values $r^{*}$ and $b^{*}$ $\operatorname{Dec}_{K_{\text {priv }}}\left(\right.$ Enc $\left._{\mathrm{K}_{\text {pub }}}\left(r^{*}, b^{*}\right)\right) \neq b^{*}$ with negligible probability. An encryption scheme is (quantum) secure if, given $\mathrm{K}_{\text {pub }}$ and Enc $_{\mathrm{K}_{\text {pub }}}\left(r^{*}, b^{*}\right)$, a (quantum) polynomial time adversary can output $b^{*}$ with at most a negligible advantage over a random guess.

We connect quantum-secure public-key encryption schemes to the hardness of learning as follows (see Theorem 5.1 for a full statement).

Result 2 Let $\mathrm{S}$ be a quantum-secure public-key cryptosystem. If $\mathcal{C}_{\mathrm{S}}$ is the concept class containing the decryption functions of the cryptosystem $\mathrm{S}$, then there is no efficient weak quantum-PAC learner for $\mathcal{C}_{\mathrm{S}}$.

The works of Kearns and Valiant [KV94] and Klivans and Sherstov [KS09] provide a connection between public-key encryption schemes and learning functions. They showed that if there exists a PAC learning algorithm for a concept class that contains the decryption function $\operatorname{Dec}_{K_{\text {priv }}}$, then it is possible to predict $b^{*}$ from $\mathrm{K}_{\mathrm{pub}}$ and $\operatorname{Enc}_{\mathrm{K}_{\mathrm{pub}}}\left(r^{*}, b^{*}\right)$ with a $1 / \operatorname{poly}(n)$ bias. They prove this by simulating the learning algorithm as follows: the distinguisher prepares examples of the form $\left(r, \operatorname{Enc}_{\mathrm{K}_{\mathrm{pub}}}(r, b)\right)$ for (uniformly) random $r$ and $b$. Using the guarantees of the classical PAC learning algorithm and the correctness of the encryption scheme, they show that the hypothesis $h$ output by the learner satisfies

$$
\underset{r^{*}, b^{*}}{\operatorname{Pr}}\left[h\left(\operatorname{Enc}_{\mathrm{K}_{\mathrm{pub}}}\left(r^{*}, b^{*}\right)\right)=b^{*}\right] \geq \frac{1}{2}+\frac{1}{n^{c}},
$$

for some $c>0$. In this paper, we quantize their argument, but the situation is much more intricate than in the classical case. Classically, $r$ and $b$ can be picked uniformly at random at each step in order to create a new training example $\left(\operatorname{Enc}_{\mathrm{K}_{\text {pub }}}(r, b), b\right)$. Quantumly, however, we do not know of an efficient way to create a quantum example $\frac{1}{\sqrt{2|\mathcal{R}|}} \sum_{r, b} \mid$ Enc $\left._{\mathrm{K}_{\mathrm{pub}}}(r, b)\right\rangle|b\rangle$, where $\mathcal{R}$ is the space of the possible randomness. Notice that a straightforward way of preparing this state involves solving the index-erasure problem [AMRR11, LR19], which is conjectured to be a hard problem to solve on a quantum computer. See Section 5 for more details. 
Instead, we first define a distribution $D$ as follows: pick poly $(n)$-many uniformly random $(r, b)$ and let $D$ be the uniform distribution over $\left\{\operatorname{Enc}_{\mathrm{K}_{\mathrm{pub}}}(r, b)\right\}$ where the set ranges over the poly $(n)$ many observed $(r, b)$. Our hope is to run the quantum learner on this distribution $D$ (which we are allowed to since we assumed that it is a quantum-PAC learner). However, we run into an issue which [KS09] need not worry about. Let Enc $_{\mathrm{K}_{\text {pub }}}\left(r^{*}, b^{*}\right)$ be the challenge string that the distinguisher needs to correctly decrypt to $b^{*}$. Observe that $\operatorname{Enc}_{\mathrm{K}_{\mathrm{pub}}}\left(r^{*}, b^{*}\right)$ need not even lie in the support of $D$, so running a quantum learner on the distribution $D$ might not even help the distinguisher in predicting $b^{*}$. In contrast, in the simulation argument of Klivans and Sherstov [KS09] the pair $\left(\operatorname{Enc}_{\mathrm{K}_{\mathrm{pub}}}\left(r^{*}, b^{*}\right), b^{*}\right)$ is always in the support of the distribution, since $r$ and $b$ are picked uniformly at random to create the classical example $\left(\operatorname{Enc}_{\mathrm{K}_{\mathrm{pub}}}(r, b), b\right)$.

Ideally, we would like to use our PAC learner on a distribution $D^{\prime}$ for which $\left(\operatorname{Enc}_{\mathrm{K}_{\mathrm{pub}}}\left(r^{*}, b^{*}\right), b^{*}\right)$ is the support of $D^{\prime}$. This would enable the distinguisher to use the guarantees of a quantum learner when run on $D^{\prime}$. The challenge here is that the distinguisher would need to find such a $D^{\prime}$ without prior knowledge of $\operatorname{Enc}_{\mathrm{K}_{\mathrm{pub}}}\left(r^{*}, b^{*}\right)$ and $b^{*}$ ! We circumvent this issue with the following observation: if two distributions are sufficiently close to each other, then the learner should perform "essentially equivalently" on both distributions. In particular, we use a training distribution $D$ that is close enough to the testing distribution $D^{\prime}$ containing $\left(\operatorname{Enc}_{\mathrm{K}_{\mathrm{pub}}}\left(r^{*}, b^{*}\right), b^{*}\right)$ so that the learning algorithm is unable to distinguish them.

We now provide more details here. Suppose we have a quantum-secure public-key cryptosystem with a (randomized) encryption function $\operatorname{Enc}_{\mathrm{K}_{\mathrm{pub}}, r}:\{0,1\} \rightarrow\{0,1\}^{n}$ and decryption function $\operatorname{Dec}_{K_{\text {priv }}}: \mathcal{E} \rightarrow\{0,1\}^{n}$, where $\mathcal{E}$ is the set of all valid encryptions and $\left(\mathrm{K}_{\text {pub }}, \mathrm{K}_{\text {priv }}\right)$ is the output of the Key-generation algorithm. Assume that the challenge string is $\operatorname{Enc}_{\mathrm{K}_{\mathrm{pub}}}\left(r^{*}, b^{*}\right)$ for a uniformly random $r^{*}, b^{*}$ and an adversary for this cryptosystem has to correctly guess $b^{*}$.

In order to construct such an adversary, first define the concept class $\mathcal{C}=\left\{\mathrm{Dec}_{\mathrm{K}_{\text {priv }}}: \mathrm{K}_{\text {priv }}\right\}$, the set of all decryption functions (one for each private key). Furthermore, assume that there is a weak quantum-PAC learner for $\mathcal{C}$ that uses $L$ examples, i.e., a polynomial-time quantum learning algorithm $\mathcal{A}$ which receives $L$ quantum examples $\sum_{x} \sqrt{D(x)}|x, c(x)\rangle$ (for an unknown distribution $D$ and concept $c \in \mathcal{C}$ ) and outputs a hypothesis $h$ that is close to the concept $c$.

As discussed previously, we now define a meaningful distribution $D$ on which the distinguisher runs the quantum learner: consider a set $S$ with $L^{3}$ tuples $\left(r_{i}, b_{i}\right)$ that are chosen uniformly at random from their respective domains; let $D$ be the uniform distribution over $\left\{\operatorname{Enc}_{\mathrm{K}_{\mathrm{pub}}}(r, b)\right.$ : $(r, b) \in S\}$. The adversary behaves as follows: run the quantum-PAC learner $\mathcal{A}$ under the distribution $D$ and provide it $L$ quantum examples of the form

$$
|\psi\rangle=\frac{1}{\sqrt{L^{3}}} \sum_{(r, b) \in S}\left|\operatorname{Enc}_{\mathrm{K}_{\mathrm{pub}}}(r, b)\right\rangle|b\rangle .
$$

When $\mathcal{A}$ outputs the hypothesis $h$, the adversary outputs $h\left(\operatorname{Enc}_{\mathrm{K}_{\mathrm{pub}}}\left(r^{*}, b^{*}\right)\right)$ as its guess for $b^{*}$. Notice that with overwhelming probability, $S$ does not contain the tuple $\left(r^{*}, b^{*}\right)$ corresponding to the challenge. So there should be no guarantee on the value of $h\left(\operatorname{Enc}_{\mathrm{K}_{\mathrm{pub}}}\left(r^{*}, b^{*}\right)\right)$. In order to overcome this, consider a quantum example state

$$
\left|\psi^{\prime}\right\rangle=\frac{1}{\sqrt{L^{3}+1}}\left(\left|\operatorname{Enc}_{\mathrm{K}_{\mathrm{pub}}}\left(r^{*}, b^{*}\right)\right\rangle\left|b^{*}\right\rangle+\sum_{(r, b) \in S}\left|\operatorname{Enc}_{\mathrm{K}_{\mathrm{pub}}}(r, b)\right\rangle|b\rangle\right) .
$$

We show that as the learner uses only $L$ quantum examples, $|\psi\rangle^{\otimes L}$, the output statistics of every quantum learning algorithm, when run on $|\psi\rangle$ and $\left|\psi^{\prime}\right\rangle$, is very similar. In fact, we show that the distribution on the hypothesis set is almost the same in each case. Now, using the performance guarantees of a quantum-PAC learning algorithm and the closeness of the distributions between 
the hypothesis sets, we conclude that $h\left(\operatorname{Enc}_{\mathrm{K}_{\text {pub }}}\left(r^{*}, b^{*}\right)\right)$ equals $b^{*}$ with probability at least $\frac{1}{2}+\frac{1}{\operatorname{poly}(n)}$. This contradicts the quantum-secure assumption on the cryptosystem.

\subsubsection{Conditional hardness of learning $\mathrm{TC}^{0}$ and $\mathrm{AC}^{0}$}

Hardness of $\mathrm{TC}^{0}$. The first consequence of Result 1 is to give a strong negative answer to the question of Aaronson [Aar05] regarding the quantum learnability of $\mathrm{TC}^{0}$ circuits, under cryptographic assumptions.

Result 3 If the Ring-Learning with Errors problem cannot be solved in quantum polynomial time, then there is no polynomial-time uniform weak quantum-PAC learner for $\mathrm{TC}^{0}$ with membership queries.

As previously mentioned, the Ring-Learning with Errors problem - i.e., the Learning with Errors problem defined over polynomial rings instead of matrices - is a cornerstone of state of the art efficient post-quantum cryptosystems and is widely believed to be hard for quantum computers. The starting point for proving Result 3 is a ring variant of the pseudo-random function family presented by Banerjee, Peikert and Rosen [BPR12], which was proven to be quantum secure by Zhandry [Zha12]. We show that our variant of the PRF is quantum secure under the assumption that the Ring-Learning with Errors (RLWE) problem cannot be solved by quantum computers efficiently. We do not define the RLWE problem here (see Section 3.2 for details), but point out that RLWE is believed to be as hard as LWE. Our variant of the PRF, denoted $\mathcal{R} \mathcal{F}$, satisfies two crucial properties: (a) every $f \in \mathcal{R F}$ can be computed by a TC ${ }^{0}$ circuit; and (b) suppose there exists a distinguisher $\mathcal{D}$ for $\mathcal{R} \mathcal{F}$ - i.e., there exists some constant $c>0$ and a polynomial-time algorithm $\mathcal{D}$ that satisfies

$$
\left|\operatorname{Pr}_{f \in \mathcal{R} \mathcal{F}}\left[\mathcal{D}^{|f\rangle}(\cdot)=1\right]-\operatorname{Pr}_{f \in \mathcal{U}}\left[\mathcal{D}^{|f\rangle}(\cdot)=1\right]\right| \geq \frac{1}{n^{c}}
$$

where $f \in \mathcal{U}$ denotes a uniformly random function - then there exists a polynomial-time algorithm that solves RLWE. We can then use our connection between PRFs and the hardness of quantum learning (Result 1) to prove Result 3.

We remark that this work contains the first explicit proof that such a PRF can indeed be implemented in $\mathrm{TC}^{0}$. Building on results laid out in [LPR13b, HAB02] for ring arithmetic, this is shown by carefully considering the cost of efficiently representing ring elements and performing operations such as iterated multiplication over these ring elements. Also, note that it is crucial to consider the RLWE version of the PRF instead of the standard LWE version as the matrix arithmetic operations needed to implement the latter would require log-depth circuits.

Given the translation between feed-forward neural networks and $\mathrm{TC}^{0}$ circuits [MSS91], our negative result on learning $\mathrm{TC}^{0}$ implies that, quantum resources do not give an exponential advantage in learning the weights of such neural networks (assuming LWE is quantum-hard).

We now make an alternate choice of parameters for the PRF $\mathcal{R} \mathcal{F}$ in order to prove a stronger hardness result on quantum learning $\mathrm{TC}^{0}$. However, a caveat of using alternate parameters is, we need to make stronger assumptions on the hardness of the RLWE problem.

Result 4 If the Ring-Learning with Errors problem cannot be solved in quantum quasi-polynomial time, then there is no quasi-polynomial time uniform weak quantum-PAC learner for $\mathrm{TC}^{0}$ with membership queries.

As far as we are aware, the above result wasn't known even in the classical literature (the only result in this flavor was proven by Fortnow and Klivans [FK09] who proved the hardness of learning based on circuit complexity theoretic assumptions). Kharitonov's proof with the BBS 
PRG [BBS86] cannot yield the above result. In fact, allowing a quasi-polynomial time learner for the BBS PRG, using Kharitonov's proof, will result in a weak-distinguisher, i.e., an algorithm that can distinguish between a random function and a PRF with $2^{- \text {polylog } n}$ probability. By contrast, our Result 1 allows us to improve upon Kharitonov's result by showing that a quasi-polynomial time learner yields a distinguisher with $1 / \operatorname{poly}(n)$ probability of distinguishing between a random function and a function from $\mathcal{R F}$.

Hardness of $A C^{0}$. Here we show how to choose a new set of parameters for the PRF $\mathcal{R F}$ that allows it to be implemented in $A C^{0}$. Unfortunately, this comes with the added costs of requiring stronger assumptions on the hardness of RLWE and weakening the achieved conclusion, namely our hardness result holds for strong learners (i.e., learners with bias $\beta \geq 1 / 6$ ). We achieve the following result.

Result 5 If there is no strongly sub-exponential time quantum algorithm for the Ring-Learning with Errors problem, then there is no $n^{\mathrm{O}\left(\log ^{v} n\right)}$-time uniform strong quantum-PAC learner for $\mathrm{AC}^{0}$ circuits on $n$ bits (for a constant $v>0$ ), using membership queries, where $d=O(\operatorname{polylog} n)$.

In order to better understand this assumption, we remark that the current best known classical algorithms for LWE require exponential time [LLL82, CN11] and any straightforward quantization of these results gives only a polynomial speedup. These algorithms are currently believed to be the best known algorithms for RLWE as well. More specifically, for $d$-dimensional RLWE instances of interest in Section 4.2, these algorithms would scale as $2^{O(\sqrt{d})}$-time. Therefore, any non-trivial improvements in the exponent for these RLWE algorithms would also be a breakthrough for the algorithms and cryptanalysis communities.

There is a key difference involved in proving Result 5, as compared to Result 3. An inherent problem in directly using the RLWR-based PRF $\mathcal{R} \mathcal{F}$ to prove Result 5 is that, any circuit computing a function $f \in \mathcal{R} \mathcal{F}$ needs to compute an iterated multiplication of many ring elements. While this can be done efficiently with a $\mathrm{TC}^{0}$ circuit, it cannot be computed using an $\mathrm{AC}^{0}$ circuit. In fact, it is a priori unclear if we can devise PRFs with super-polynomially large truth tables whose hardness is based on a quantum-hard problem while also being computable in $\mathrm{AC}^{0} .^{9}$

In order to overcome this issue, we use a technique that was also used by Kharitonov [Kha93]; by considering PRFs with a truth-table size that is sub-exponential in the dimension of the underlying RLWR problem. Specifically, we obtain a PRF $\mathcal{R} \mathcal{F}^{\prime}$ whose truth-table size is polynomial in $n$ while the dimension, $d$, is poly-logarithmic in $n$. We show that computing an arbitrary function $f \in \mathcal{R F}^{\prime}$ can be performed by an $\mathrm{AC}^{0}$ circuit. However, this increase in truth table size - from being super-polynomial in $d$ as in the $\mathrm{TC}^{0}$ case to being sub-exponential in $d$ for $\mathrm{AC}^{0}-$ which means that a stronger hardness assumption is needed to guarantee the security of this PRF. To this end, by assuming that $d$-dimensional RLWE cannot be solved by quantum computers in strongly sub-exponential time (i.e., $2^{O\left(d^{1 / \eta}\right)}$-time for every constant $\eta>2$ ), the PRF that we consider here is quantum-secure. With this new PRF such that $d=O(\operatorname{polylog} n)$, we repeat the arguments of Result 3 and show the $n^{O\left(\log ^{v} n\right)}$-time hardness of quantum learning for $\mathrm{AC}^{0}$ for a constant $v>0$ that depends on $\eta$.

We remark that Result 5 matches the upper bound of Linial, Mansour and Nisan [LMN93] (up to the constant $v$ in the exponent), who showed how to classically learn $\mathrm{AC}^{0}$ circuits on $n$ bits using $n^{O(\operatorname{polylog}(n))}$ many random examples $(x, c(x))$ where $x$ is drawn from the uniform distribution. Our

\footnotetext{
${ }^{9}$ Here we mean that the size of the truth table for each function in the PRF is super-polynomial in the dimension of the underlying quantum problem.
} 
result shows that, up to the $v$ in the exponent, the classical learning algorithm of [LMN93] is optimal even if one is given access to uniform quantum examples and is allowed to make quantum queries, under the RLWE assumption explained previously.

It is worth noting that our result also implies conditional hardness results for classical learning of $\mathrm{TC}^{0}$ and $A C^{0}$, under the assumption that solving RLWE is hard for classical computers (instead of factoring in the case of Kharitonov [Kha93]).

\subsubsection{Conditional hardness of PAC learning $\mathrm{TC}_{2}^{0}$}

Our third hardness result is the following.

Result 6 If the Learning with Errors problem cannot be solved in quantum polynomial time, then there is no polynomial-time weak quantum-PAC learner for $\mathrm{TC}_{2}^{0}$.

In contrast to our first hardness result for $\mathrm{TC}^{0}$, we stress that the quantum learners in this result are quantum-PAC learners. The main idea to prove this result is to consider the LWE-based publickey cryptosystem proposed by Regev [Reg09] (see Section 3.1.2). Klivans and Sherstov [KS09] considered this cryptosystem and showed that the decryption functions in this cryptosystem can be implemented by circuits in $\mathrm{TC}_{2}^{0}$. We can then use our connection between quantum learning and quantum-secure cryptosystems (Result 2) to derive Result 6.

\subsection{Open questions}

This work raises a number of interesting open questions, which we list below.

Learning $A C_{d}^{0}$. What is the smallest $d$ for which we can prove that quantum learning $\mathrm{AC}_{d}^{0}$ is (conditionally) hard? Bshouty and Jackson [BJ99] gave a quantum polynomial-time algorithm for $A C_{2}^{0}$ and for some universal constant $d^{\prime} \geq 3$ (independent of the input-size of the $A C_{d^{\prime}}^{0}$ circuit), our work rules out polynomial-time learning algorithms for $A C_{d^{\prime}}^{0}$, assuming there exists no subexponential time quantum algorithm for the RLWE problem. Classically, using the constant-depth construction of PRGs by Naor and Reingold [NR04] in Kharitonov's [Kha93] result, one can show that $\mathrm{AC}_{5}^{0}$ is hard to learn (assuming factoring is hard on a classical computer).

Uniform learning of $\mathrm{TC}_{2}^{0}$. The conditional hardness results of $\mathrm{TC}^{0}$ under the uniform distribution and hardness of quantum-PAC learning $\mathrm{TC}_{2}^{0}$ do not rule out the possibility that $\mathrm{TC}_{2}^{0}$ admits polynomial-time quantum learning algorithms under the uniform distribution. It is an open question to show if $\mathrm{TC}_{2}^{0}$ can be learned quantum-efficiently under the uniform distribution or if we can show a conditional hardness result.

Hardness of (quantum) learning quantum shallow circuits Linial et al. [LMN93] showed the quasi-polynomial time learnability of shallow classical circuits. Correspondingly, what would be the time/sample complexity of learning shallow quantum circuits with a quantum learner? Recently Chung and Lin [CL18] showed that polynomially many samples suffice to learn the class of polynomial-sized quantum circuits.

Hardness of quantum learning from other assumptions Finally, we leave as an open question the possibility of proving the hardness of quantum learning from other complexity-theoretic assumptions. We now point to some potential directions: 
- Recent results have proved Strong Exponential Time Hypothesis (SETH)-based hardness of the Gap-SVP [AS18] problem and the Subset - sum [ABHS19] problem (actually, they prove the limit of a specific approach to solve the Subset - sum problem). These hardness results do not imply any hardness for learning problems directly and we wonder if they can be tightened in order to make it possible.

- Oliveira and Santhanam [OS17] established a connection between learning theory, circuit lower bounds and pseudo-randomness. Is it possible to quantize such connections?

- Daniely and Schwartz [DS16] showed a complexity-theoretic hardness of PAC learning DNFs. Could we also show hardness for quantum-PAC learning DNFs as well?

\subsection{Differences with previous version}

This version of this work differs from the previous version, due to a mistake in the former proof. We discuss the main change below.

In the previous version of this work, we claimed to prove hardness of learning $\mathrm{TC}^{0}$ and $\mathrm{AC}^{0}$ based on the quantum security of pseudorandom generators. In our proof, we assumed that the run-time of a learning algorithm depended only on the input size. Essentially, we considered a learning algorithm $\mathcal{A}$ that runs in time $n^{a}$ with bias $n^{-c}$ for a concept class on $n$ bits, and showed the existence of a PRG $G$ that takes an $n$-bit seed having a stretch $n^{d}$ where $d>a+c$ that $\mathcal{A}$ cannot learn efficiently.

However, in the standard definition of polynomial-time learnability, the run-time of a quantum learning algorithm should depend both on the input size $n$ as well as the representation size of the concept class. In other words, an efficient learner $\mathcal{A}$ for a PRG $G$, with an $n$-bit seed, stretch $n^{d}$ and representation size $n^{s}$, is allowed to take time $O\left(\operatorname{poly}\left(n, n^{s}\right)\right)$. Under this definition, for every instantiation of our PRG, since $n^{s}>n^{d}$, there exists an efficient learning algorithm with run-time $n^{a}>n^{s}>n^{d}$, thereby not leading to any contradiction, or hardness-of-learning the concept class.

In order to achieve our hardness results in this updated version, we use pseudorandom functions such that for every choice of parameters, their truth-table size $T(n) \gg \operatorname{poly}(n, s(n))$, where $s(n)$ is the representation size of the function. Hence for every learning algorithm $\mathcal{A}$ running in time $t(n)$, we show the existence of a PRF with truth table size $T(n) \gg O(\operatorname{poly}(n, s(n)) \geq t(n)$ that $\mathcal{A}$ cannot learn efficiently.

\section{Organization}

In Section 2, we state some required lemmas, discuss cryptographic primitives and formally define the classical and quantum learning models. In Section 3, we discuss the (Ring)Learning with Errors problem and its variants along with the pseudo-random functions constructed from these problems. In Section 4, we describe the connection between quantum-secure PRFs and quantum learning under the uniform distribution and prove our main results showing the hardness of learning $\mathrm{TC}^{0}$ and $\mathrm{AC}^{0}$. Finally, in Section 5, we show the connection between quantum-secure public-key cryptosystems and quantum-PAC learning and conclude with the proof for the hardness of PAC-learning $\mathrm{TC}_{2}^{0}$.

\section{Acknowledgments}

S.A. thanks Andrea Rocchetto and Ronald de Wolf for discussions during the initial stage of this work and Robin Kothari for introducing him to this problem. S.A. and A.S. did part of this work 
during the Workshop on Quantum Machine Learning at the Joint Centre for Quantum Information and Computer Science, University of Maryland. S.A. did part of this work at QuSoft, CWI and was supported by ERC Consolidator Grant QPROGRESS. We thank Anurag Anshu, Gorjan Alagic, Daniel Apon and Prabhanjan Ananth for helpful discussions. We thank Robin Kothari and Ronald de Wolf for many helpful comments which improved the presentation of this paper. S. A. thanks Aravind Gollakota and Daniel Liang for useful discussions. We thank Oded Regev for noticing a mistake in a previous version of this paper. We are grateful to Leo Ducas for helping us find a suitable set of parameters for our Ring-LWE hardness assumptions.

\section{Preliminaries}

\subsection{Notation and basic claims}

We define some widely used definitions below. For the set of integers $\mathbb{Z}$ and an integer $q$, define $\mathbb{Z}_{q}:=\mathbb{Z} / q \mathbb{Z}$, i.e., $\mathbb{Z}_{q}$ is the set of integers $(\bmod q)$. For $k \in \mathbb{N}$, we let $[k]:=\{0, \ldots, k-1\}$. All logarithms will be taken with respect to the base 2 . A function $\mu: \mathbb{N} \rightarrow \mathbb{R}$ is said to be negligible in a parameter $\lambda \geq 1$, which we denote $\operatorname{negl}(\lambda)$, if it satisfies the following:

for every integer $t>0$, there exists an integer $K_{t}>0$ such that for all $\lambda>K_{t}$, we have $|\mu(\lambda)|<\lambda^{-t}$. Similarly, a function $\eta: \mathbb{N} \rightarrow \mathbb{R}$ is said to be non-negligible in $\lambda$ if there exists an integer $t>0$ and $K_{t}>0$ such that $\eta(\lambda) \geq \lambda^{-t}$ for all $\lambda>K_{t}$. For a distribution $D:\{0,1\}^{n} \rightarrow[0,1]$, we write $x \sim D$ to say that $x$ is drawn according to the distribution $D$. For simplicity, we say that a function $f(n)=\operatorname{poly}(n)$, if there exist constants $a, b>0$ such that $n^{a}<f(n)<n^{b}$.

An algorithm $\mathcal{A}$ is said to have oracle access to a function $f$, which is denoted by $\mathcal{A}^{f}$, if $\mathcal{A}$ is allowed to (classically) query $f(x)$ for every $x$ in the domain of $f$ with unit computational cost. When $\mathcal{A}$ is a quantum algorithm, it is said to have quantum oracle access to $f$, denoted by $\mathcal{A}^{|f\rangle}$, if $\mathcal{A}$ is allowed to perform the operation $\sum_{x} \alpha_{x, b}|x\rangle|b\rangle \rightarrow \sum_{x, b} \alpha_{x, b}|x\rangle|f(x) \oplus b\rangle$ with unit computational cost for every $x \in\{0,1\}^{n}, b \in\{0,1\}$ and $\alpha_{x, b} \in \mathbb{C}$

We say there is a non-negligible advantage in distinguishing $D$ from another distribution $D^{\prime}$ if there is a $\operatorname{poly}(n)$-time adversary Adv (i.e., algorithm) such that

$$
\left|\operatorname{Pr}_{x_{1}, \ldots, x_{L} \sim D}\left[\operatorname{Adv}\left(x_{1}, \ldots, x_{L}\right)=1\right]-\operatorname{Pr}_{x_{1}, \ldots, x_{L} \sim D^{\prime}}\left[\operatorname{Adv}\left(x_{1}, \ldots, x_{L}\right)=1\right]\right| \geq \eta(n),
$$

where $\eta(n)$ is a non-negligible function and $L$ is a polynomial in $n$.

Analogously, we say that there is a non-negligible advantage in distinguishing the functions $f, f^{\prime}: \mathcal{X} \rightarrow \mathcal{Y}$ if there is a poly $(n)$-time adversary Adv (i.e., algorithm) that has oracle access to $f$ or $f^{\prime}$ such that

$$
\left|\operatorname{Pr}\left[\operatorname{Adv}^{f}()=1\right]-\operatorname{Pr}\left[\operatorname{Adv}^{f^{\prime}}()=1\right]\right| \geq \eta(n),
$$

where $\eta(n)$ is a non-negligible function. Here $n$ is some security parameters and $|\mathcal{X}|,|\mathcal{Y}|$ scale with $n$. If Adv instead has quantum oracle access to a function $f$, we denote it by $\operatorname{Adv}|f\rangle$.

\subsection{Information theory and communication complexity}

We describe some basic concepts in information theory that we use later. Given a probability distribution $D: \mathcal{X} \rightarrow[0,1]$, the entropy of a random variable $X \sim D$ is given by

$$
\mathbf{H}(X)=-\sum_{X \in \mathcal{X}} \operatorname{Pr}_{X \sim D}[X=x] \log \left(\operatorname{Pr}_{X \sim D}[X=x]\right) .
$$


The binary entropy of $\varepsilon \in[0,1]$ is defined as $\mathbf{H}_{b}(\varepsilon)=-\varepsilon \log \varepsilon-(1-\varepsilon) \log (1-\varepsilon)$. Moreover, $\mathbf{H}_{b}(\varepsilon)$ can be upper bounded as follows.

Fact 2.1 For all $\varepsilon \in[0,1 / 2]$ we have the binary entropy $\mathbf{H}_{b}(\varepsilon) \leq O(\varepsilon \log (1 / \varepsilon))$, and from the Taylor series expansion of $\mathbf{H}_{b}(\varepsilon)$, we have

$$
1-\mathbf{H}_{b}(1 / 2+\varepsilon) \leq 2 \varepsilon^{2} / \ln 2+O\left(\varepsilon^{4}\right)
$$

Given a probability distribution $D: \mathcal{X} \times \mathcal{Y} \rightarrow[0,1]$, and the random variables $(X, Y) \sim D$, the conditional entropy of $X$ given $Y$ is

$$
\mathbf{H}(X \mid Y)=-\sum_{x \in \mathcal{X}, y \in \mathcal{Y}} \operatorname{Pr}_{(X, Y) \sim D}[(X, Y)=(x, y)] \log \left(\frac{\operatorname{Pr}_{(X, Y) \sim D}[(X, Y)=(x, y)]}{\operatorname{Pr}_{X \sim D}[X=x]}\right) .
$$

Given a probability distribution $D: \mathcal{X} \times \mathcal{Y} \times \mathcal{Z} \rightarrow[0,1]$, the random variables $(X, Y, Z) \sim D$, the conditional mutual information between $X$ and $Y$ given $Z$ is

$$
\mathbf{I}(X: Y \mid Z)=\mathbf{H}(X \mid Z)-\mathbf{H}(X \mid Y, Z) .
$$

The following fact about conditional entropy and prediction errors will be useful for us.

Lemma 2.2 (Fano's inequality) Let $X$ be a random variable taking values in $\{0,1\}$ and $Y$ be a random variables taking values in $\mathcal{Y}$. Let $f: \mathcal{Y} \rightarrow\{0,1\}$ be a prediction function, which predicts the value of $X$ based on an observation of $Y$. Suppose $\varepsilon=\operatorname{Pr}[f(Y) \neq X]$ is the probability of error made by the prediction function, then $\mathbf{H}(X \mid Y) \leq \mathbf{H}_{b}(\varepsilon)$.

We now briefly describe communication complexity. For details, we refer the reader to [Tou15, KLGR16]. Here, we are interested in the setup with two parties Alice (denoted $A$ ) and Bob (denoted $B$ ). $A$ (resp. $B$ ) receives input $X$ (resp. $Y$ ) such that $(X, Y) \sim D$ for a publicly known probability distribution $D$. $A$ and $B$ then follow some protocol $\pi$ in which they exchange quantum information back-and-forth. Finally, $B$ outputs a random variable $Z$. The quantum communication complexity of the protocol QCC $(\pi)$ is the number of qubits communicated in the protocol $\pi$.

Touchette [Tou15] defined the notion of quantum information complexity for a protocol, denoted QIC $(\pi)$, which is rather subtle and out of the scope of this work. In [Tou15, Theorem 1], Touchette showed that for all protocols $\pi$, we have $\operatorname{QIC}(\pi) \leq \operatorname{QCC}(\pi)$. Similarly, Kerenidis et al. [KLGR16, Theorem 1] showed that QIC $(\pi)$ is at most the classical information complexity of the protocol $\mathrm{CIC}(\pi)$, whose definition we omit here. Also, it is not hard to see that if $B$ outputs some value $Z$, then

$$
\mathbf{I}(Z: X \mid Y) \leq \mathrm{CIC}(\pi)
$$

Putting together [Tou15, Theorem 1] and [KLGR16, Theorem 1] along with the inequality above, we obtain the following corollary.

Corollary 2.3 Given a quantum communication protocol $\pi$ between two parties $A$ and $B$ whose inputs are $X$ and $Y$, respectively, drawn from a distribution $D$. Let $Z$ be the output of $B$. Then,

$$
\mathbf{I}(Z: X \mid Y) \leq Q C C(\pi) \text {. }
$$

\subsection{Cryptographic primitives}

Definition 2.4 (Pseudo-random functions) Let $n$ be security parameter, $\mathcal{X}, \mathcal{K}$ and $\mathcal{Y}$ be finite sets and $\mathcal{F}=\left\{f_{\mathbf{k}}: \mathcal{X} \rightarrow \mathcal{Y}\right\}$ be family of functions indexed by $\mathbf{k} \in \mathcal{K} . \mathcal{F}$ is called a pseudo-random function family if, for every polynomial-time probabilistic algorithm Adv and for every constant $c>0$ we have

$$
\left|\operatorname{Pr}_{f_{\mathrm{k}} \in \mathcal{F}}\left[\operatorname{Adv}^{f_{\mathrm{k}}}(\cdot)=1\right]-\operatorname{Pr}_{g \in \mathcal{U}}\left[\operatorname{Adv}^{g}(\cdot)=1\right]\right|<\frac{1}{s^{c}},
$$

where $f_{\mathbf{k}}$ is picked uniformly at random from $\mathcal{F}$ and $g$ is picked uniformly at random from $\mathcal{U}$, the set of all functions from $\mathcal{X}$ to $\mathcal{Y}$. 
In this work, we also consider quantum polynomial-time distinguishers. In short, when we say that a pseudo-random function is secure, we mean that it satisfies Definition 2.4 and it is quantum-secure if it satisfies a variation of Definition 2.4 where Adv is a polynomial-time quantum algorithm and has quantum query access to the functions $f_{\mathbf{k}}$ and $g$. We point out that such a distinction is important. For example, there are examples of PRFs that are secure against (quantum) adversaries with classical oracle access to the function, but that are not secure when the adversary is allowed to perform quantum queries $\left[\mathrm{BDF}^{+} 11\right]$.

\subsection{Learning models}

\subsubsection{Classical distribution-independent learning}

We begin by introducing the classical Probably Approximately Correct (PAC) model of learning which was introduced by Leslie Valiant [Val84]. A concept class $\mathcal{C}$ is a collection of Boolean functions $c:\{0,1\}^{n} \rightarrow\{0,1\}$, which are often referred to as concepts. In the PAC model, a learner $\mathcal{A}$ is given access to a random example oracle $\operatorname{EX}(c, D)$ where $c \in \mathcal{C}$ is an unknown target concept (which the learner is trying to learn) and $D:\{0,1\}^{n} \rightarrow[0,1]$ is an unknown distribution. At each invocation of $\operatorname{EX}(c, D)$ the oracle returns a labelled example $(x, c(x))$ where $x$ is drawn from the distribution $D .{ }^{10}$ Then $\mathcal{A}$ outputs a hypothesis $h$ and we say that $\mathcal{A}$ is an $(\varepsilon, \delta)-P A C$ learner for a concept class $\mathcal{C}$ if it satisfies the following:

for every $\varepsilon, \delta \in[0,1]$, for all $c \in \mathcal{C}$ and distributions $D$, when $\mathcal{A}$ is given $\varepsilon, \delta$ and access to the $\operatorname{EX}(c, D)$ oracle, with probability $\geq 1-\delta, \mathcal{A}$ outputs a hypothesis $h$ such that

$$
\operatorname{Pr}_{x \sim D}[h(x) \neq c(x)] \leq \varepsilon
$$

The learner's advantage over a random guess is given by $\beta=\frac{1}{2}-\frac{\varepsilon}{2}$ and $2 \beta=1-\varepsilon$ is called the bias of the learner.

The sample complexity of $\mathcal{A}$ is the maximum number of invocations of the $\operatorname{EX}(c, D)$ oracle which the learner makes when maximized over all $c \in \mathcal{C}$ and all distributions $D$. Finally the $(\varepsilon, \delta)$-PAC sample complexity of $\mathcal{C}$ is defined as the minimum sample complexity over all $\mathcal{A}$ that $(\varepsilon, \delta)$-PAC learn $\mathcal{C}$. The time complexity of $(\varepsilon, \delta)$-PAC learning $\mathcal{C}$ is the minimum number of time steps of an algorithm $\mathcal{A}$ that $(\varepsilon, \delta)$-PAC learns $\mathcal{C}$ (where the minimum is over all $\mathcal{A}$ that $(\varepsilon, \delta)$-PAC learn $\mathcal{C}$ ). We say that $\mathcal{A}(\varepsilon, \delta)$-weakly learns (resp. strongly learns) $\mathcal{C}$ if $\varepsilon=\frac{1}{2}-n^{-c}$ (resp. $\varepsilon=1 / 3$ ) for some constant $c>0$ and input size $n$. Freund et al. [FSA99] showed that weak-PAC learning $\mathcal{C}$ is equivalent to strong-PAC learning.

\subsubsection{Quantum distribution-independent learning}

The quantum model of PAC learning was introduced by Bshouty and Jackson [BJ99]. Instead of having access to an $\operatorname{EX}(c, D)$ oracle, here a quantum-PAC learner has access to a $\mathrm{QEX}(c, D)$ oracle

$$
\operatorname{QEX}(c, D):\left|0^{n}, 0\right\rangle \rightarrow \sum_{x} \sqrt{D(x)}|x, c(x)\rangle,
$$

and we leave the QEX $(c, D)$ oracle undefined on other basis states. We refer to the state produced by $\operatorname{QEX}(c, D)$ as a quantum example, which is a coherent superposition over classical labeled examples. A quantum-PAC learner is given access to copies of quantum examples and performs a POVM (positive-valued-operator measurement), where each outcome of the POVM corresponds

\footnotetext{
${ }^{10}$ Note that the oracle $\operatorname{EX}(c, D)$ doesn't take any input and simply returns a labelled example.
} 
to a hypothesis. Similar to the classical distribution-independent learning setting, the quantum sample complexity of an algorithm $\mathcal{A}$ is the maximum number of invocations of the $\mathrm{QEX}(c, D)$ oracle which the learner makes, when maximized over all $c \in \mathcal{C}$ and all distributions $D$. The $(\varepsilon, \delta)$ quantum PAC sample complexity of $\mathcal{C}$ is defined as the minimum quantum sample complexity over all $\mathcal{A}$ that $(\varepsilon, \delta)$-quantum-PAC learn $\mathcal{C}$.

\subsubsection{Uniform distribution learning}

The classical PAC model of learning places a strong requirement on learners, i.e., the learner needs to $(\varepsilon, \delta)$-PAC learn $\mathcal{C}$ for every unknown distribution $D$. In classical learning theory, there has been a lot of work in understanding a weaker model of learning - when $D$ is restricted to the uniform distribution on $\{0,1\}^{n}$ (which we denote as $\mathcal{U}$ ). In this restricted model, a classical learner is given access to $\operatorname{EX}(c, \mathcal{U})$ (known to the learner) which generates $(x, c(x))$ where $x$ is sampled according to the uniform distribution $\mathcal{U}$. An algorithm $\mathcal{A}$ is said to $(\varepsilon, \delta)$-learn $\mathcal{C}$ under $\mathcal{U}$ if it satisfies the following:

for every $\varepsilon, \delta \in[0,1]$, for all $c \in \mathcal{C}$, when $\mathcal{A}$ is given $\varepsilon, \delta$ and access to the $\operatorname{EX}(c, \mathcal{U})$ oracle, with probability $\geq 1-\delta, \mathcal{A}$ outputs a hypothesis $h$ such that $\operatorname{Pr}_{x \sim \mathcal{U}}[h(x) \neq c(x)] \leq \varepsilon$

The sample complexity and time complexity of learning $\mathcal{C}$ under the uniform distribution is defined similar to Section 2.4.1 when we fix $D=\mathcal{U}$.

One can similarly consider the case when a quantum learner is given access to $\mathrm{QEX}(c, \mathcal{U})$

$$
\operatorname{QEX}(c, \mathcal{U}):\left|0^{n}, 0\right\rangle \rightarrow \frac{1}{\sqrt{2^{n}}} \sum_{x}|x, c(x)\rangle .
$$

We leave QEX undefined on other basis states and assume the learner does not have access to the inverse of $\operatorname{QEX}(c, \mathcal{U})$. The quantum sample complexity and time complexity of learning a concept class $\mathcal{C}$ under the uniform distribution is defined similar to Section 2.4.2 when we restrict $D=\mathcal{U}$. A powerful advantage of being given access to QEX $(c, \mathcal{U})$ is Fourier sampling. We do not introduce Fourier sampling here and refer the interested reader to [AW17, Section 2.2.2].

\subsubsection{Learning with membership queries}

The classical model of PAC learning places yet another strong requirement on learners, i.e., the learner is only given access to labelled examples generated by the oracle $\operatorname{EX}(c, D)$ (for an unknown $c \in \mathcal{C}$ and distribution $D$ ). Angluin [Ang87] relaxed this requirement and introduced the model of learning with membership queries. In this scenario, in addition to $\operatorname{EX}(c, D)$, a learner is given access to a membership oracle $\mathrm{MQ}(c)$ for the unknown target concept $c$, which takes as input $x \in\{0,1\}^{n}$ and returns $c(x)$. An algorithm $\mathcal{A}$ is said to $(\varepsilon, \delta)$-learn $\mathcal{C}$ under $D$ with membership queries if it satisfies the following:

for every $\varepsilon, \delta \in[0,1]$, for all $c \in \mathcal{C}$, when $\mathcal{A}$ is given $\varepsilon, \delta$ and access to $\operatorname{EX}(c, D), \mathrm{MQ}(c)$ oracles, with probability $\geq 1-\delta, \mathcal{A}$ outputs a hypothesis $h$ such that $\operatorname{Pr}_{x \sim D}[h(x) \neq c(x)] \leq \varepsilon$.

We abuse notation by saying the sample complexity of $\mathcal{A}$ is the maximum number of invocations of an oracle ${ }^{11}$ when maximized over all $c \in \mathcal{C}$ under distribution $D$. The sample complexity and time complexity of learning $\mathcal{C}$ under $D$ given membership queries is defined similar to Section 2.4.1 (where the classical learner now has access to $\mathrm{MQ}(c)$ in addition to $\mathrm{EX}(c, D)$ ).

\footnotetext{
${ }^{11}$ Note that an invocation of either $\operatorname{EX}(c, D)$ or $\mathrm{MQ}(c)$ counts as one application of the oracle.
} 
One can similarly consider the case when a quantum learner is additionally given access to a quantum membership query oracle $\mathrm{QMQ}(c)$

$$
\mathrm{QMQ}(c):|x, b\rangle \rightarrow|x, b \oplus c(x)\rangle,
$$

for $x \in\{0,1\}^{n}$ and $b \in\{0,1\}$. The quantum learner is allowed to perform arbitrary unitary operations in between applications of the QMQ $(c)$ oracle. The quantum sample complexity and time complexity of learning $\mathcal{C}$ under $D$ given quantum membership queries is defined similar to Section 2.4.2 (where the classical learner now has access to $\mathrm{QMQ}(c)$ in addition to $\mathrm{QEX}(c, D)$ ). In this paper, we will also view the concept as as $c \in\{0,1\}^{2^{n}}$, described by the truth-table of $c:\{0,1\}^{n} \rightarrow\{0,1\}$. In this case, we say a learning algorithm is allowed to make queries to a string $c \in\{0,1\}^{N}$, it means that the algorithm is given access to the oracle QMQ $(c):|x, b\rangle \rightarrow\left|b \oplus c_{x}\right\rangle$ for $x \in[N]$ and $b \in\{0,1\}$.

Learning with membership queries under the uniform distribution. Finally, one can combine the learning models in this section with Section 2.4.3 and consider (quantum) learners which are given access to (quantum) membership queries and (quantum) labelled examples when the underlying distribution $D$ is restricted to the uniform distribution $\mathcal{U}$. We say $\mathcal{A}$ is an $(\varepsilon, \delta)$-uniform PAC learner for $\mathcal{C}$ with membership queries if $\mathcal{A}(\varepsilon, \delta)$-learns $\mathcal{C}$ under $\mathcal{U}$ with membership queries. Similarly we can define a $(\varepsilon, \delta)$-uniform quantum-PAC learner for $\mathcal{C}$ with quantum membership queries. In this paper we will consider such learners in the weak and strong learning settings, and for simplicity we will omit the $(\varepsilon, \delta)$-dependence when referring to the classical-PAC or quantumPAC learners. The sample complexity and time complexity of such learners is defined similar to Section 2.4.1, 2.4.2 (wherein the classical learner now has access to $\operatorname{EX}(c, \mathcal{U})$ and $\mathrm{MQ}(c)$ and the quantum learner has access to $\operatorname{QEX}(c, \mathcal{U})$ and $\mathrm{QMQ}(c))$. In order to further understand the theoretical aspects of quantum machine learning, we refer the reader to [SP18, $\left.\mathrm{AAD}^{+} 15, \mathrm{AW} 17\right]$.

\subsection{Circuits and neural networks}

In this paper we will be concerned with the class of shallow or constant-depth Boolean circuits that consist of AND, OR, NOT and Majority gates. We define these classes formally now.

\subsubsection{The circuit classes $\mathrm{AC}^{0}$ and $\mathrm{TC}^{0}$}

An $\mathrm{AC}^{0}$ circuit on $n$ bits consists of AND, OR and NOT gates whose inputs are $x_{1}, \ldots, x_{n}, \overline{x_{1}}, \ldots, \overline{x_{n}}$. Fan-in to the AND and OR gates are unbounded. The size of the circuit (i.e., the number of gates in the $A C^{0}$ circuit) is bounded by a polynomial in $n$ and the depth of the circuit is a constant (i.e., independent of $n$ ). Furthermore, the gates at level $i$ of the circuit have all their inputs coming from the $(i-1)$-th level and all the gates at the same level are AND or OR gates. The class of Boolean functions that can be expressed by such a depth- $d$ circuit is written as $A C_{d}^{0}$ and $A C^{0}=\bigcup_{d \geq 0} A C_{d}^{0}$. A depth- $d$ threshold circuit, denoted $\mathrm{TC}_{d}^{0}$, is similar to an $\mathrm{AC}_{d}^{0}$ circuit, except that the circuit is also allowed to have Majority gates $\operatorname{MAJ}:\{0,1\}^{n} \rightarrow\{0,1\}$, where $\operatorname{MAJ}(x)=1$ if and only if $\sum_{i} x_{i} \geq n / 2$.

Additionally, we will need the following definition of halfspaces and polynomial threshold functions in order to discuss about depth- $2 \mathrm{TC}_{2}^{0}$ circuits. A half-space in $n$ dimensions is a Boolean function $f:\{0,1\}^{n} \rightarrow\{0,1\}$ of the form

$$
f(x)=\llbracket \sum_{i} a_{i} x_{i} \geq \theta \rrbracket
$$

where $a_{1}, \ldots, a_{n}$ and $\theta$ are fixed integers and $\llbracket \cdot \rrbracket$ denotes the indicator function which evaluates to 1 if and only if $\sum_{i} a_{i} x_{i} \geq \theta$. The intersection of $k$ half-spaces is a function of the form $g(x)=\bigwedge_{i=1}^{k} f_{i}(x)$ 
where the $f_{i}$ s are half-spaces and $\wedge$ is the AND function. A polynomial threshold function (PTF) of degree $d$ is a Boolean function of the form $f(x)=\llbracket p(x) \geq 0 \rrbracket$, where $p$ is a degree- $d$ polynomial with integer coefficients. Note that a half-space is a degree-1 PTF. A PTF $f$, defined as $f(x)=\llbracket p(x) \geq 0 \rrbracket$, is called light if the sum of the absolute value of the coefficients in $p$ is at most polynomial in $n$. Using this, we now have the following claim.

Claim 2.5 A polynomially-light halfspace on $n$ dimensions can be computed in $\mathrm{TC}_{2}^{0}$.

Proof. First observe that each half-space on $n$ bits is already a majority gate (with the inputs suitably negative and replicated based on the coefficients $\left.a_{1}, \ldots, a_{n}\right)$ and the top gate $\operatorname{AND}\left(f_{1}, \ldots, f_{t}\right)$ can be replaced by the majority gate $\operatorname{MAJ}\left(-t, f_{1}, \ldots, f_{t}\right)$, which is a depth- 2 threshold circuit.

Neural networks and $\mathrm{TC}^{0}$. One motivation for learning the concept class $\mathrm{TC}^{0}$ is that this class is a theoretical way to model neural networks. Although we do not deal with neural networks in this paper, we briefly mention their connection to $\mathrm{TC}^{0}$ circuits. A feed-forward neural network can be modeled as an acyclic directed graph where the nodes consist of neurons. ${ }^{12}$ Every neuron is associated with some internal weights $w_{0}, \ldots, w_{n} \in \mathbb{R}$. The action of the neuron is defined as follows: it takes as real input $x_{1}, \ldots, x_{n}$ and generates an output signal $y$ defined by

$$
y=\sigma\left(\sum_{i \in[n]} w_{i} x_{i}-w_{0}\right),
$$

where $\sigma$ is a transfer function. The size of a neural network is the number of neurons in the network and the depth of the network is the number of layers in the network between the input and final output. A commonly used transfer function is the sigmoid function, defined as $\sigma(t)=\left(1+e^{-t}\right)^{-1}$. The sigmoid function is a continuous approximation of the threshold function $\operatorname{Thr}_{\mathrm{w}_{0}, \mathrm{w}_{1}, \ldots, \mathrm{w}_{\mathrm{n}}}$, which on input $x_{1}, \ldots, x_{n}$, outputs 1 if $\sum_{i} w_{i} x_{i} \geq w_{0}$ and 0 otherwise. Maass, Schnitger and Sontag [MSS91] showed an equivalence between feed-forward neural networks and $\mathrm{TC}^{0}$ circuits. Before we state their theorem, we first define the following:

Definition 2.6 Let $\varepsilon>0$ and $f:\{0,1\}^{n} \rightarrow\{0,1\}$ be a Boolean function. We say a feed-forward neural network $C \varepsilon$-computes $f$, if there exists a $t_{C} \in \mathbb{R}$ such that: on input $x \in\{0,1\}^{n}, C(x) \geq t_{C}+\varepsilon$ if $f(x)=1$ and $C(x) \leq t_{C}-\varepsilon$ if $f(x)=0$.

We now state the main result of [MSS91].

Theorem 2.7 (Theorem 4.1[MSS91]) Let $d \geq 1$. Let $\mathrm{T}_{d}$ be the class of functions $f:\{0,1\}^{n} \rightarrow\{0,1\}$ such that there exists a depth-d feed-forward neural network $C$ on $n$ bits, whose size is poly $(n)$, the individual weights in $C$ have absolute value at most poly $(n)$ and $C \frac{1}{3}$-computes $f$. Then, $\mathrm{T}_{d}=\mathrm{TC}_{d}^{0} \cdot{ }^{13}$

In particular, this theorem implies that Boolean functions that are computable by constantdepth polynomial-sized neural networks with neuron-weights bounded by some polynomial in the input size, can be implemented as a circuit in $\mathrm{TC}^{0}$. So an alternate definition of $\mathrm{TC}^{0}$ is the class of constant-depth neural networks with polynomial size and weights bounded polynomially in the input length.

\footnotetext{
${ }^{12}$ In general, neural networks are cyclic directed graphs. In order to make the connection to $\mathrm{TC}^{0}$ we $\operatorname{consider}^{\mathrm{a}}$ subclass of neural networks called feed-forward neural-networks.

${ }^{13}$ The $1 / 3$ can be replaced by an arbitrary constant independent of $n$.
} 


\section{Hardness assumptions and cryptographic constructions}

In this section we give a detailed introduction to lattice-based problems and some cryptographic primitives built using them. Our motivation in doing this, firstly, is to highlight the subtleties inherent in the hardness results and security proofs associated with these lattice-based problems and primitives respectively. Secondly, the details also help in explaining the subtleties that are reflected in our utilization of these primitives for showing the hardness of quantum learning.

\subsection{Learning with Errors (LWE)}

Throughout this section, we will need the following notation. Let $\chi$ be a distribution over $\mathbb{Z}$ and assume that we can efficiently sample from $\chi \cdot{ }^{14}$ Let $B>0$. We say that $\chi$ is $B$-bounded if $\operatorname{Pr}_{e \sim \chi}[|e|>B] \leq \mu(n)$, where $e, B$ are at most $n$ bits long and $\mu$ is a function that is negligible in $n$. In other words, a distribution $\chi$ is $B$-bounded if, with high probability, the magnitude of $e$ when drawn according to $\chi$ is at most $B$. Also, a discrete Gaussian refers to the standard normal Gaussian distribution on the real line which is restricted to take integer values. Now, we can define the decision version of the Learning with Errors (LWE) problem.

Definition 3.1 $\left(\operatorname{LWE}_{d, q, \chi, m}\right)$ The (decision) Learning with Errors problem with dimension $d$, modulus $q$ and a B-bounded distribution $\chi$ over $\mathbb{Z}$ is defined as follows: On input $m$ independent samples $\left\{\left(\mathbf{a}_{i}, b_{i}\right) \in \mathbb{Z}_{q}^{d} \times \mathbb{Z}_{q}\right\}_{i}$, where the $\mathbf{a}_{i}$ are uniformly sampled from $\mathbb{Z}_{q}^{d}$, distinguish (with non-negligible advantage) between the following two cases:

- (LWE-samples) The $b_{i} s$ are noisy products with respect to a fixed secret $\mathbf{s}$ distributed uniformly in $\mathbb{Z}_{q}^{d}$, i.e., $b_{i}$ s are of the form $b_{i}=\mathbf{a}_{i} \cdot \mathbf{s}+e_{i}(\bmod q)$ where $e_{i} \in \mathbb{Z}$ is sampled according to $\chi$ conditioned on $\left|e_{i}\right| \leq B$.

- (Uniform samples) For every $i, b_{i}$ is uniformly sampled from $\mathbb{Z}_{q}$ and is independent of $\mathbf{a}_{i}$.

When the number of samples $m$ is arbitrary (i.e., not bounded in terms of the dimension $d$ ), we will simply denote the problem as $\operatorname{LWE}_{d, q, \chi}$.

Consider an efficient distinguisher $\mathcal{D}$ as defined in Definition 2.4 that distinguishes a distribution $D$ from being uniformly random. Then, if $D$ is the distribution generated by LWE-samples, the distinguisher $\mathcal{D}$ would clearly serve as an efficient algorithm to solve the (decision) LWE problem. We formally define this below.

Definition 3.2 (Distinguishers for $\mathrm{LWE}_{d, q, \chi, m}$ ) An algorithm $\mathcal{D}$ is a distinguisher for the (decision) $\mathrm{LWE}_{d, q, \chi, m}$ problem if, given $m$ independent samples from $\mathbb{Z}_{q}^{d} \times \mathbb{Z}_{q}$, it distinguishes $m$ LWE-samples $\left\{\mathbf{a}_{i}, b_{i}^{(1)}\right\}_{i}$ from $m$ uniformly random samples $\left\{\mathbf{a}_{i}, b_{i}^{(2)}\right\}_{i}$ with non-negligible advantage i.e., there exists $a$ non-negligible function $\eta: \mathbb{N} \rightarrow \mathbb{R}$ such that

$$
\mid \operatorname{Pr}_{\left(\mathbf{a}_{i}, b_{i}^{(1)}\right)}\left[\mathcal { D } ( \{ \mathbf { a } _ { i } , b _ { i } ^ { ( 1 ) } \} _ { i } ) \text { outputs 1]- } \underset { ( \mathbf { a } _ { i } , b _ { i } ^ { ( 2 ) } ) } { \operatorname { P r } } \left[\mathcal{D}\left(\left\{\mathbf{a}_{i}, b_{i}^{(2)}\right\}_{i}\right) \text { outputs 1] } \mid \geq \eta(d) .\right.\right.
$$

When $\mathcal{D}$ is a poly $(d, m)$-time probabilistic (resp. quantum) algorithm, it is said to be an efficient classical (resp. quantum) distinguisher.

\footnotetext{
${ }^{14}$ The distribution $\chi$ is dependent on input size $n$, but we drop the $n$-dependence for notational simplicity.
} 


\subsubsection{Hardness of LWE}

For a suitable choice of parameters, it is believed that LWE is a hard problem to solve. This is based on the worst-case hardness of lattice-based problems such as GapSVP $\gamma$ (decision version of the shortest vector problem) or SIVP (shortest independent vectors problem) [Reg09, Pei09]. We do not introduce these problems or discuss their hardness here; an interested reader can refer to Peikert's survey [Pei16] on the topic.

The first hardness result for LWE was a quantum reduction to the GapSVP problem [Reg09, Pei09]. Subsequent works succeeded in de-quantizing the reduction from [Reg09] except that the modulus $q$ was required to be super-polynomial in the dimension $d$ [Pei09]. Later, Brakerski et al. $\left[\mathrm{BLP}^{+} 13\right]$ built on this result and other techniques from fully homomorphic encryption, obtaining a reduction with a polynomial modulus.

Theorem 3.3 (Hardness of LWE $\left.\left[\mathrm{BLP}^{+} 13\right]\right)$ Let $d, q \geq 1$ and $\alpha \in(0,1)$ be such that $q=\operatorname{poly}(d)$ and $\alpha q \geq 2 d$. Let $D_{\mathbb{Z}_{q}, \alpha}$ be the discrete Gaussian distribution over $\mathbb{Z}_{q}$ with standard deviation $\alpha q$. Then there exists a classical reduction from the worst-case hardness of the d-dimensional GapSVP $\widetilde{O}(d / \alpha)$ problem to the $\mathrm{LWE}_{d^{2}, q, D_{\mathbb{Z}_{q}, \alpha}}$ problem.

The corollary below provides a suitable choice for parameters that lead to hard LWE instances.

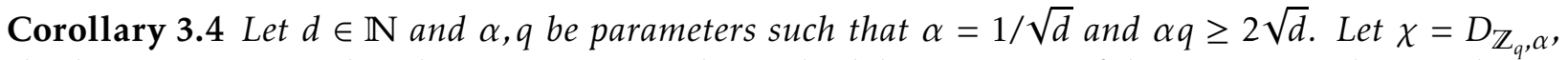
the discrete Gaussian distribution over $\mathbb{Z}_{q}$ with standard deviation $\alpha q$. If there exists a polynomial-time quantum distinguisher for $\mathrm{LWE}_{d, q, \chi, m}$, then there exists a polynomial-time quantum algorithm for the $\sqrt{d}$-dimensional GapSVP $\widetilde{O}\left(d^{1.5}\right)$ problem.

It is believed that there exists no polynomial-time quantum algorithms for the $d$-dimensional

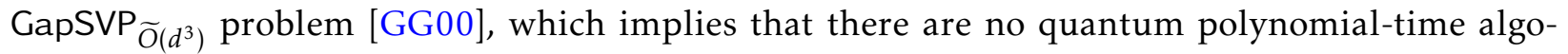
rithms for LWE with the parameters as stated in Corollary 3.4.

We would like to stress that we show hardness results for learning constant-depth circuits with quantum examples, based on the hardness of solving LWE with classical samples. This does not contradict the work by Grilo et al. [GKZ19] who prove that LWE is easy when quantum examples are provided.

\subsubsection{Public-key encryption scheme based on LWE}

In this section we describe the public-key cryptosystem proposed by Regev [Reg09] whose security is based on the hardness of LWE.

Definition 3.5 (LWE - PKE [Reg09]) The LWE - PKE $d, q, m$ public-key encryption scheme consists of:

Key-generation: Pick $s \in \mathbb{Z}_{q}^{d}$ and $A \in \mathbb{Z}_{q}^{m \times d}$ uniformly at random from the respective supports. Draw $e \in \mathbb{Z}_{q}^{d}$ from the distribution $\chi$, as defined in the LWE problem. Let $\boldsymbol{b}=$ As $+e$ and output $\mathrm{K}_{\mathrm{priv}}=\boldsymbol{s}$ and $\mathrm{K}_{\mathrm{pub}}=(A, b)$.

Encryption: To encrypt a bit c using $\mathrm{K}_{\mathrm{pub}}=(A, \boldsymbol{b})$, pick $S \subseteq[\mathrm{m}]$ uniformly at random and the encryption is $\left(\mathbf{1}_{S}^{T} \cdot A, \mathbf{1}_{S}^{T} \cdot \boldsymbol{b}+c\left\lfloor\frac{q}{2}\right\rfloor\right)$, where $\mathbf{1}_{S} \in\{0,1\}^{m}$ is defined as $\mathbf{1}_{S}(i)=1$ if and only if $i \in S$

Decryption: In order to decrypt the ciphertext $(a, b)$ using $\mathrm{K}_{\text {priv }}=s$, output 0 if $b-a^{T} s(\bmod q) \leq\left\lfloor\frac{q}{4}\right\rfloor$, otherwise output 1.

Theorem 3.6 Let $d \in \mathbb{N}, \varepsilon>0$ be a constant, $q=\operatorname{poly}(d)$ and $m \geq(1+\varepsilon)(d+1) \log q$ be polynomially bounded in $d$. Then, the probability of decryption error for $\mathrm{LWE}-\mathrm{PKE}_{d, q, m}$ is $2^{-\omega\left(d^{2} / m\right)}$. Moreover, an 
adversary can distinguish an encryption of 0 from an encryption of 1 in polynomial time with nonnegligible advantage over a random guess iff there is a polynomial-time distinguisher for $\mathrm{LWE}_{d, q, \chi, m}$.

The following property of LWE - PKE was proven by Klivans and Sherstov [KS09].

Lemma 3.7 (Lemma 4.3 [KS09]) The decryption function of LWE - PKE can be computed by light degree-2 PTFs.

\subsection{Ring-LWE}

One of the main drawbacks of LWE-based cryptographic primitives is that their representations are not compact, which makes them inefficient to implement. To overcome this, Lyubashevsky et al. [LPR13a] proposed a variant of LWE over polynomial rings, called Ring-LWE. Unfortunately, the efficiency of implementing Ring-LWE does not come for free: first, the average-case hardness of Ring-LWE is currently supported only by the worst-case hardness of problems on ideal lattices; and secondly the reduction is still (partly) quantum (see Section 3.2.1 for further discussion).

The following notation will be used whenever we refer to the ring variants of lattice problems. Let $R$ be a degree- $d$ cyclotomic ring of the form $R:=\mathbb{Z}[X] /\left\langle X^{d}+1\right\rangle$ where $d$ is a power of 2 . In particular, the elements of $R$ will be represented by their residues modulo $\left(X^{d}+1\right)-$ which are integer polynomials of degree less than $d$. For an integer modulus $q$, we let $R_{q}:=R / q R=\mathbb{Z}_{q}[X] /\left\langle X^{d}+1\right\rangle$ where its elements are canonically represented by integer polynomials of degree less than $d$ with coefficients from $\mathbb{Z}_{q}$.

Let $\Upsilon$ denote an efficiently sampleable distribution over the elements of $R$ that is concentrated on elements having $B$-bounded integer coefficients, i.e., let $\Upsilon$ be a variant of the $d$-dimensional discrete Gaussian distribution where each coefficient of the integer polynomial (in some canonical representation) is $B$-bounded with overwhelmingly high probability. As with LWE, the Ring-LWE problem focuses on finding a secret ring element $\mathbf{s} \in R_{q}$ when given noisy ring products $(\mathbf{a}, \mathbf{a} \cdot \mathbf{s}+e)$ as samples where $\cdot$ and + refer to the multiplication and addition operations in $R_{q}$.

Definition $3.8\left(\operatorname{RLWE}_{d, q, \Upsilon, m}\right)$ The (decision) Ring Learning with Errors problem with dimension $d$, modulus $q$ and $a$ B-bounded distribution $\Upsilon$ over $R$ is defined as follows: On input $m$ independent samples $\left\{\left(\mathbf{a}_{i}, b_{i}\right) \in R_{q} \times R_{q}\right\}_{i}$, where the $\mathbf{a}_{i}$ are uniformly sampled from $R_{q}$, distinguish (with non-negligible advantage) between the following two cases:

- (RLWE-samples) The $b_{i}$ s are noisy products with respect to a fixed secret $\mathbf{s}$ distributed uniformly in $R_{q}$, i.e., $b_{i}$ s are of the form $b_{i}=\mathbf{a}_{i} \cdot \mathbf{s}+e_{i}(\bmod q)$ where $e_{i} \in R$ is sampled according to $\Upsilon$.

- (Uniform samples) For every $i, b_{i}$ is uniformly sampled from $R_{q}$ and is independent of $\mathbf{a}_{i}$.

Note that, similar to Definition 3.2, we can also consider distinguishers, $\mathcal{D}$, for the ring-LWE problem $\operatorname{RLWE}_{d, q, \Upsilon, m}$. At times, we will consider the normal form of the problem where the secret is sampled from the error distribution modulo $q$ i.e., $s \leftarrow \chi^{d}(\bmod q)$. It is known, from [LPR13b], that this form of the problem is as hard as the problem in Definition 3.8.

\subsubsection{Hardness of Ring-LWE}

Lyubashevsky, et. al. [LPR13a] first showed a worst-case hardness reduction from the shortest vector problem $\mathrm{SVP}_{\gamma}$ on a class of structured lattices called ideal lattices to Ring-LWE. It is believed that $\mathrm{SVP}_{\gamma}$ is hard in the worst case, even for quantum algorithms. For instance, the best 
known quantum algorithms for (general) $\mathrm{SVP}_{\text {poly }(d)}$ still run in time $2^{O(d)}$. Although the hardness reduction between Ring-LWE and $\mathrm{SVP}_{\gamma}$ uses ideal lattices, the hardness assumptions for $\mathrm{SVP}_{\text {poly }}(d)$ are still fairly standard. One caveat here is that that reduction poses restrictions on the types of rings and requires that the modulus $q=\operatorname{poly}(d)$. For more on this subject, we direct the interested reader to Peikert's survey [Pei16].

Subsequently, Langlois and Stehlé [LS15] improved the hardness result for any modulus $q$ with poly $(d)$ bit-size (therefore $q$ can be super-polynomial in $d$ ). More recently, Peikert et al. [PRS17] further improved the hardness result to work for every ring and modulus by obtaining a direct reduction from the worst-case hardness of the $\operatorname{SIVP}_{\gamma}$ (find approximately short independent vectors in a lattice of length within a $\gamma$-factor of the optimum) on ideal lattices to the (decision) $\operatorname{RLWE}_{d, q, \Upsilon, m}$ problem. We use the following result from their work applied to cyclotomic rings.

Theorem 3.9 (Quantum Reduction from [PRS17]) Let $R$ be a degree-d cyclotomic ring and let $m=$ $\operatorname{poly}(d)$. Let $\alpha=\alpha(d) \in(0,1)$ and $q=q(d) \geq 2$ be an integer such that $\alpha \leq 1 / 2 \sqrt{\log d / d}$ and $\alpha q \geq \omega(1)$. Let $\Upsilon$ be a d-dimensional discrete Gaussian distribution over $R$ with standard deviation at most $\alpha q$ in each coefficient. Then, there exists a polynomial-time quantum reduction from the d-dimensional $\mathrm{SIVP}_{\gamma}$ over $R$ to the $\operatorname{RLWE}_{d, q, \Upsilon, m}$ problem for any $\gamma \leq \max \{\omega(\sqrt{d \log d} / \alpha), \sqrt{2} d\}$.

When $\gamma=\widetilde{O}(\operatorname{poly}(d))$, the best known quantum algorithms for $\operatorname{SIVP}_{\gamma}$ are still expected to take exponential time and any sub-exponential time algorithm for these approximation factors will be considered a breakthrough in the cryptography and quantum community. Based on this, we will use the following three assumptions on the hardness of RLWE in the remainder of this work.

Assumption 1 (Polynomial time hardness) Let $0<\tau<1, d \in \mathbb{N}, q=2^{O\left(d^{\tau}\right)}, r \leq \operatorname{poly}(d)$ and $\Upsilon(r)$ be the discrete Gaussian distribution over degree-d cyclotomic rings with standard deviation at most $r$ in each coefficient. The $\mathrm{RLWE}_{d, q, \Upsilon(r)}$ problem is hard for a poly $(d)$-time quantum computer.

Assumption 1 is used in Corollary 4.5 to show that $\mathrm{TC}^{0}$ cannot be learned in polynomial time.

Assumption 2 (Quasi-polynomial time hardness) Let $0<\tau<1, d \in \mathbb{N}, q=2^{O\left(d^{\tau}\right)}, r \leq \operatorname{poly}(d)$ and $\Upsilon(r)$ be the discrete Gaussian distribution over degree-d cyclotomic rings with standard deviation at most $r$ in each coefficient. The $\operatorname{RLWE}_{d, q, \Upsilon(r)}$ problem is hard for a $2^{O(\text { polylog(d)) }}$-time quantum computer.

Assumption 2 helps to show that $\mathrm{TC}^{0}$ cannot be learned in quasi-polynomial time in Corollary 4.5.

Assumption 3 (Strongly sub-exponential time hardness) Let $0<\tau<1,0<\varepsilon<\frac{1}{2}, d \in \mathbb{N}, q=$ $2^{O\left(d^{\tau}\right)}, r \leq d^{\lambda}$ for $0<\lambda<1 / 2-\varepsilon$ and $\Upsilon(r)$ be the discrete Gaussian distribution over degree- $d$ cyclotomic rings with standard deviation at most $r$ in each coefficient. For every constant $\eta>2$, the $\operatorname{RLWE}_{d, q, \Upsilon(r)}$ problem is hard for a $2^{o\left(d^{1 / \eta}\right)}$-time quantum computer.

Assumption 3 is used in Corollary 4.7 to show that $\mathrm{AC}^{0}$ cannot be learned in polynomial time.

\subsection{Learning with Rounding (LWR)}

While the hardness results for LWE and RLWE suggest that they are good candidates for quantumsecure cryptographic primitives, the amount of randomness these schemes need to generate samples make them unsuitable for the construction of pseudo-random functions. For this reason, we consider the Learning with Rounding problem (LWR) which was introduced by Banerjee, Peikert and Rosen [BPR12] and can be seen as a deterministic variant of LWE. 
The following notation will be used in this section. For $\mathbf{a}, \mathbf{s} \in \mathbb{Z}_{q}^{d}$, let a.s be defined as $\sum_{j \in[d]} a_{j} s_{j}$ $\bmod q$. For $q \geq p \geq 2$, we define $\lfloor\cdot\rceil_{p}: \mathbb{Z}_{q} \rightarrow \mathbb{Z}_{p}$ as $\lfloor x\rceil_{p}=\lfloor(p / q) \cdot x\rceil$ where $\lfloor\cdot\rceil$ denotes the closest integer. Additionally, when $\mathbf{x}$ is a ring element in $R_{q}$, we extend this rounding operation to rings as follows: define $\left\lfloor\cdot T_{p}: R_{q} \rightarrow R_{p}\right.$ as the operation where each polynomial coefficient $x_{i} \in \mathbb{Z}_{q}$ of $\mathbf{x}$ is rounded to $\mathbb{Z}_{p}$ as $\left\lfloor x_{i}\right\rceil_{p}$ respectively. From here onwards, we assume that $q$ has a poly $(d)$ bit size, i.e. $\log q \leq O(\operatorname{poly}(d))$, and, without loss of generality, that $p$, and $q$ are powers of 2 .

Definition $3.10\left(\mathrm{LWR}_{\boldsymbol{d}, \boldsymbol{q}, \boldsymbol{p}, \boldsymbol{m}}\right)$ The (decision) Learning with Rounding problem with dimension $d$, modulus $q$ and rounding modulus $p<q$ is defined as follows: on input $m$ independent samples $\left\{\left(\mathbf{a}_{i}, b_{i}\right) \in\right.$ $\left.\mathbb{Z}_{q}^{d} \times \mathbb{Z}_{p}\right\}_{i}$ where the $\mathbf{a}_{i}$ 's are sampled uniformly from $\mathbb{Z}_{q}^{d}$, distinguish (with non-negligible advantage) between the following two cases:

- (LWR-samples) There is a fixed secret $\mathbf{s}$ uniformly sampled from $\mathbb{Z}_{q}^{d}$ such that the $b_{i}$ s are rounded products with respect to $\mathbf{s}$, i.e., $b_{i}=\left\lfloor\mathbf{a}_{i} \cdot \mathbf{s}\right\rceil_{p}$ for every $i$.

- (Uniform samples) For every $i, b_{i}$ is uniformly sampled from $\mathbb{Z}_{p}$ and is independent of $\mathbf{a}_{i}$.

As LWR inherits all issues of inefficient implementation exhibited by LWE, we are particularly interested in the ring version of this problem namely, Learning with Rounding over Rings. This is defined below.

Definition $3.11\left(\operatorname{RLWR}_{d, q, p, m}\right)$ The (decision) Learning with Rounding problem with dimension $d$, modulus $q$ and rounding modulus $p<q$ is defined as follows: on input $m$ independent samples $\left\{\left(\mathbf{a}_{i}, \mathbf{b}_{i}\right) \in\right.$ $\left.R_{q} \times R_{p}\right\}_{i}$ where the $\mathbf{a}_{i}$ 's are sampled uniformly from $R_{q}$, distinguish (with non-negligible advantage) between the following two cases:

- (RLWR-samples) There is a fixed secret $\mathbf{s}$ uniformly sampled from $R_{q}$ such that the $b_{i}$ s are rounded ring products with respect to $\mathbf{s}$, i.e., $\mathbf{b}_{i}=\left\lfloor\mathbf{a}_{i} \cdot \mathbf{s}\right\rceil_{p}$ for every $i$.

- (Uniform samples) For every $i, b_{i}$ is uniformly sampled from $R_{p}$ and is independent of $\mathbf{a}_{i}$.

While the definition above samples a secret uniformly from $R_{q}$, an equivalent problem samples $s$ using the RLWE error distribution $\Upsilon(\bmod q)$. This will have a bearing when we consider pseudo-random functions constructed from RLWR. When the number of samples is arbitrary, the corresponding problems are denoted as $\mathrm{LWR}_{d, q, p}$ and $\mathrm{RLWR}_{d, q, p}$. Similar to Definition 3.2 of distinguishers for $\operatorname{LWE}_{d, q, \chi, m}$ one could analogously define classical and quantum distinguishers for the $\operatorname{LWR}_{d, q, p, m}$ and $\mathrm{RLWR}_{d, q, p, m}$ problems.

\subsubsection{PRFs from the LWR problem}

The hardness reduction in [BPR12] from $\operatorname{RLWR}_{d, q, p, m}\left(\right.$ resp. $\operatorname{LWR}_{d, q, p, m}$ ) to $\operatorname{RLWE}_{d, q, \Upsilon}$ (resp. $\operatorname{LWE}_{d, q, \chi}$ ) for $q=$ superpoly $(d)$ implies that these problems may be hard for quantum computers. This makes them good candidates from which to construct quantum-secure PRFs. The following PRF construction from [BPR12, Section 5] will be of importance to us.

Definition 3.12 (RLWE degree- $k$ PRF) For parameters $d \in \mathbb{N}$, moduli $q \geq p \geq 2$ and input length $k \geq 1$, let $R$ be a degree-d cyclotomic ring. The RLWE-PRF $F_{d, q, p, k}$ function family $\mathcal{R} \mathcal{F}$ is defined as $\mathcal{R F}:=\left\{f_{\mathbf{a}, \mathbf{s}_{1}, \ldots, \mathbf{s}_{k}}:\{0,1\}^{k} \rightarrow R_{p}\right\}$ where $\mathbf{a} \in R_{q}, \mathbf{s}_{i} \in R_{q}$ for $i \in[k]$ and

$$
f_{\mathbf{a}, \mathbf{s}_{1}, \ldots, \mathbf{s}_{k}}(x):=\left\lfloor\mathbf{a} \cdot \Pi_{i \in[k]} \mathbf{s}_{i}^{x_{i}}\right\rceil_{p}
$$

where $\lfloor\cdot\rceil_{p}$ denotes the rounding procedure over ring elements. 
Clearly, every function in the family $\mathcal{R} \mathcal{F}$ defined above, outputs a ring element from $R_{p}$. However, for the purposes of constructing the simplest concept class that is hard to learn, we would prefer a PRF that outputs a single bit. We can achieve this by modifying the construction to output the most significant bit of the PRFs output from Definition 3.12 and denote it as the One-Bit RLWR PRF (1B-RPRF) as defined below.

Definition 3.13 (One-Bit RLWR PRF) For parameters $d \in \mathbb{N}$ and moduli $q \geq p \geq 2$, let $R$ be a degree$d$ cycolotomic ring. Let $\phi_{p}: R_{q} \rightarrow\{0,1\}$ be defined as $\phi_{p}(\mathbf{g})=\operatorname{msb}\left(\lfloor\mathbf{g}\rceil_{p}\right)$ where $\mathrm{msb}(\cdot)$ for a ring element denotes the most significant bit of it's first polynomial coefficient. For an input length $k \geq$ 1 , the $1 \mathrm{~B}-\mathrm{RPRF}_{d, q, p, k}$ function family $\mathcal{R} \mathcal{F}$ is defined as $\mathcal{R} \mathcal{F}:=\left\{f_{\mathbf{a}, \mathbf{s}_{1}, \ldots, \mathbf{s}_{k}}:\{0,1\}^{k} \rightarrow\{0,1\}\right\}$ where $\mathbf{a}, \mathbf{s}_{1}, \ldots, \mathbf{s}_{k} \in R_{q}$ and

$$
f_{\mathbf{a}, \mathbf{s}_{1}, \ldots, \mathbf{s}_{k}}(x)=\phi_{p}\left(\mathbf{a} \cdot \Pi_{i \in[k]} \mathbf{s}_{\mathbf{i}}^{x_{i}}\right) .
$$

Note that the definition above is equivalent for $\phi$ outputting any arbitrary fixed bit from the representation of the ring element. [BPR12] showed that the RLWE degree- $k$ PRFs are both secure against classical adversaries. Combining techniques from [BPR12] and [Zha12], ${ }^{15}$ one can conclude in a fairly straightforward manner that the RLWE degree- $k$ PRF is also secure against quantum adversaries under the RLWE assumption. For completeness, we show how this security proof also implies the security of the One-Bit RLWR PRFs.

Lemma 3.14 If the RLWE degree- $k$ PRF is quantum-secure, then so is the One-Bit RLWR PRF.

Proof. Let us assume that there exists a distinguisher $\mathcal{D}$ for the One-Bit RLWR PRF. This means that $\mathcal{D}$ can make (quantum) queries to an oracle $O$ and can distinguish if $O$ is a function picked from the One-Bit RLWR PRF family or a uniformly random Boolean function.

We construct a distinguisher $\mathcal{D}^{\prime}$ for the RLWE degree- $k$ PRF that uses $\mathcal{D}$ as a sub-routine. By definition, $\mathcal{D}^{\prime}$ has (quantum) query access to an oracle $O^{\prime}$ that is either a function picked from the RLWE degree- $k$ PRF family or a uniformly random function that outputs an element from $R_{p}$.

In order to use $\mathcal{D}$ as a sub-routine successfully, $\mathcal{D}^{\prime}$, using queries to its oracle $O^{\prime}$, simulates an oracle $\widetilde{O}$ that will answer the (quantum) queries from $\mathcal{D}$. In this case, $\widetilde{O}$ is a function from OneBit RLWR PRF when $O^{\prime}$ is a function from RLWE degree- $k$ PRF, or a uniformly random Boolean function otherwise. Using the definition of $\widetilde{O}$ and the fact that $\mathcal{D}$ is a distinguisher for the One-Bit RLWR PRF, one can conclude that $\mathcal{D}^{\prime}$ is indeed a distinguisher for the RLWE degree- $k$ PRF.

We finish the proof by showing how $\mathcal{D}^{\prime}$ can simulate $\widetilde{O}$ using queries to $O^{\prime}$. We now describe the action of $\widetilde{O}$ on the query $|x\rangle|y\rangle$ made by $\mathcal{D}^{\prime}$ - without loss of generality, it suffices to define the actions of $\widetilde{O}$ the basis states since as the operations of $\mathcal{D}^{\prime}$ are unitary. $\mathcal{D}^{\prime}$ makes a call to $O^{\prime}$ using a $|x\rangle$ as the input register and fresh ancilla bits set to $|0\rangle$ as the output register, resulting in $|x\rangle|y\rangle|z\rangle$, where $z=O^{\prime}(x)$. $\mathcal{D}^{\prime}$ computes the state $|x\rangle\left|y \oplus z_{1}\right\rangle|z\rangle$, where $z_{1}$ denotes the first bit of $z$, followed by a second query to $O^{\prime}$ using the first and third registers as the input and output registers, respectively. $\mathcal{D}^{\prime}$ then discards the last register (which has value $|0\rangle$ ) and answers the query with $|x\rangle\left|y \oplus z_{1}\right\rangle$. Notice that if $O^{\prime}$ is an oracle to the RLWE degree- $k$ PRF family, then $\widetilde{O}$ is simulating a function from the One-Bit RLWR PRF family; and if $O^{\prime}$ is a random function, the first bit of the representation of its output is a random bit, which ensures that $\widetilde{O}$ is also a random function.

Putting all the security proofs together, we obtain the choice of parameters that would make some One-Bit RLWR PRF family quantum secure.

\footnotetext{
${ }^{15}$ Zhandry [Zha12] proved that the LWE degree- $k$ PRF is also secure against quantum adversaries. This was done by showing that the classical security of the PRF under the LWE assumption implies the quantum security under the same assumption.
} 
Lemma 3.15 Let $d \in \mathbb{N}, r>0,2 \leq p \ll q$ and $k=\omega(\log d)$ such that $\log q \leq O(\operatorname{poly}(d))$.

Let $R$ be a degree-d cyclotomic ring and let $\Upsilon(r)$ be the d-dimensional discrete Gaussian distribution over $R$ with standard deviation at most $r$ in each coefficient. Let $q \geq p \cdot k(r \sqrt{d+k} \cdot \omega(\log d))^{k} \cdot d^{\omega(1)}$. Let $\mathcal{R F}$ be the $1 \mathrm{~B}-\mathrm{RPRF}_{d, q, p, k}$ function family where each secret $\mathbf{s}_{i}$ is independently drawn from $\Upsilon(r)$. If $\mathrm{RLWE}_{d, q, \Upsilon(r)}$ is hard for an $t(n)$-time quantum computer, then $\mathcal{R} \mathcal{F}$ is a quantum-secure pseudo-random function family against $O(t(n))$ adversaries.

Proof Sketch. We begin with the security proof for the RLWE-PRF $d, q, p, k$ against classical adversaries as shown in [BPR12, Section 5]. By making adjustments almost identical to those in Zhandry's proof [Zha12, Section F] that was used to prove the quantum security of the LWE base PRF, we conclude that the RLWE- $\mathrm{PRF}_{d, q, p, k}$ is secure against quantum adversaries. Using Lemma 3.14, we obtain the parameters $q, p, k$ for which the $1 \mathrm{~B}-\mathrm{RPRF}_{d, q, p, k}, \mathcal{R F}$ is secure against quantum adversaries.

Next, we demonstrate that there exist efficient constant-depth circuit implementations for the One-Bit RLWR PRF family $\mathcal{R} \mathcal{F}$.

Lemma 3.16 Let $d \in \mathbb{N}, k=\omega(\log d)$, let $q=d^{\omega(1)}$ and $2 \leq p \leq q$ be powers of 2 such that $\log q \leq$ $O(\operatorname{poly}(d))$. Let $R$ be the degree-d cyclotomic ring. Consider the $1 \mathrm{~B}-\mathrm{RPRF}_{d, q, p, k}$ function family $\mathcal{R} \mathcal{F}$ defined in Definition 3.13. For every $\mathbf{a}, \mathbf{s}_{1}, \ldots, \mathbf{s}_{k} \in R_{q}, f_{\mathbf{a}, \mathbf{s}_{1}, \ldots, \mathbf{s}_{k}} \in \mathcal{R} \mathcal{F}$ can be computed by an $O(\operatorname{poly}(d, k))-$ sized $\mathrm{TC}^{0}$ circuit.

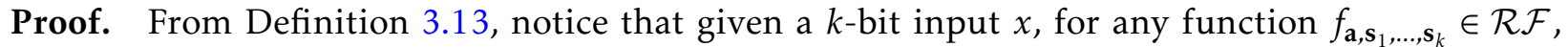
$f_{\mathbf{a}, \mathbf{s}_{1}, \ldots, \mathbf{s}_{k}}(x)$ can be computed as follows: (a) take the product of a with those secrets $\mathbf{s}_{i}$ for which $x_{i}=1$ to obtain an element in $R_{q}$, (b) round it to an element in $R_{p}$ and (c) output the first bit of the representation of this element. Since $q$ and $p$ are powers of 2 , the coefficient-wise rounding procedure just truncates each coefficient to the $\log p$ most significant bits. Hence, the last two steps can be efficiently executed by a poly $(d)$-sized $T C^{0}$ circuit as there are most $d$ coefficients to round. It remains to show that computing the iterated multiplication of at most $k+1$ ring elements can be performed using a $\mathrm{TC}^{0}$ circuit.

Recall that the ring elements, in the standard representation are stored as degree- $d$ polynomials and multiplication in this representation may not be efficient. However, using a canonical embedding $\sigma: R_{q} \rightarrow \mathbb{C}^{d}$, arising from algebraic number theory, any ring element $z \in R_{q}$ can be mapped to the complex vector $\left(z\left(\eta_{i}\right)\right)_{i} \in \mathbb{C}^{d}$ where $\eta_{i}$ is the $i$ th complex root of $(-1)$. In other words, $z$ can be efficiently stored as complex vectors of length $d$ and each vector entry of size at most $O(\log q)=O($ poly $d)$ bits. The advantage in this representation is that ring multiplication reduces to coefficient wise multiplication of these vectors [LPR13a, LPR13b]. Now, Step (a) can be broken up into the following operations:

1. Convert the $k+1$ ring elements $\mathbf{a}, \mathbf{s}_{1}, \ldots, \mathbf{s}_{k}$ into their canonical embeddings $\sigma(\mathbf{a}),\left\{\sigma\left(\mathbf{s}_{i}\right)\right\}_{i \in[k]} \in \mathbb{C}^{d}$.

2. Perform coordinate wise product of at most $k+1$-vectors in this embedding.

3. Convert the solution back into the standard representation, i.e., perform the inverse of the embedding $\sigma$

The first and last steps to convert to and from the canonical embedding is performed using Fast Fourier Transforms (FFT) and its variants or the Chinese Remainder Representation (CRR) [LPR13b] which can be executed in $\mathrm{TC}^{0}$ [RT92, HAB02]. The second step is just $d$-parallel 
iterated multiplications of at most $k+1$ elements each of $O(\log q)=O($ poly $d)$-bits which can be performed by an $O(\operatorname{poly}(d, k))$-sized TC ${ }^{0}$ circuit [HAB02]. Putting all the steps together, $f_{\mathbf{a}, \mathbf{s}_{1}, \ldots, \mathbf{s}_{k}}(x)$ can be computed by an $O(\operatorname{poly}(d, k))$-sized $\mathrm{TC}^{0}$ circuit.

One problem that prevents us from computing any function from the family $\mathcal{R} \mathcal{F}$ in smaller circuit classes, say $\mathrm{AC}^{0}$, lies in the fact that performing FFT, the CRR embedding and iterated multiplication cannot be done efficiently in $\mathrm{AC}^{0}$. However, we use two observations to demonstrate that there exist sub-exponential sized $A C^{0}$ circuits that compute functions in the family $\mathcal{R F}$ : (i) arithmetic operations on $O($ polylog $n)$-bit numbers can be executed using poly $(n)$-sized $\mathrm{AC}^{0}$ circuits $^{16}$ [MT98]; (ii) iterated multiplication of $t$ numbers each of length at most $n$ bits can be performed in poly $\left(2^{n^{\varepsilon}}, 2^{t^{\varepsilon}}\right)$-sized $\mathrm{AC}^{0}$ circuits [HAB02, HV06]. We proceed by defining the PRF with a smaller dimension in the underlying problem such that the PRF's circuit size scales sub-exponentially in the dimension. This resembles the technique used by Kharitonov [Kha93] to show that some instances of the BBS PRG can be implemented in $\mathrm{AC}^{0}$.

Lemma 3.17 Let $d \in \mathbb{N}$ and choose constants $c>\eta>1$ and $\varepsilon<1$ such that $\varepsilon \leq \frac{\eta}{\eta+c}$. Let $d>2$ and $k=(d)^{\frac{1}{\eta}}$. Choose $2<p \ll q=\operatorname{superpoly}(d)$ as powers of 2 such that $\log q=\frac{k^{2}}{k+1} \log d$. Consider the $1 \mathrm{~B}-\mathrm{RPRF}_{d, q, p, k}$ family $\mathcal{R} \mathcal{F}$ defined in Definition 3.13. For every $\mathbf{a}, \mathbf{s}_{1}, \ldots, \mathbf{s}_{k} \in R_{q}, f_{\mathbf{a}, \mathbf{s}_{1}, \ldots, \mathbf{s}_{k}} \in \mathcal{R} \mathcal{F}$ can be computed by a $2^{O\left(d^{1 / c}\right)}$-sized $\mathrm{AC}^{0}$ circuit of depth $\mathrm{O}(1 / \varepsilon)$.

Proof. For notational simplicity, we set $d^{\prime}=2^{(d)^{1 / c}}$ i.e., $d=\log ^{c} d^{\prime}$ and proceed by first calculating the circuit size in terms of $d^{\prime}$. The proof follows the ideas used in the proof of Lemma 3.16. Specifically, the operations performed to evaluate $f_{\mathbf{a}, \mathbf{s}_{1}, \ldots, \mathbf{s}_{k}}(x)$ break up in the same manner as before. Also, note that the input to the circuit is determined by the $k$-bit string $x$.

Let us start our analysis with the rounding of some element from $R_{q}$ to $R_{p}$. This can be done by truncating each coefficient to the most significant $\log p$ bits where $\log p \ll \log q=\frac{k^{2}}{k+1} \log d \leq$ $k \log d=O\left(\right.$ polylog $\left.d^{\prime}\right)$ since $d=\log ^{c} d^{\prime}$ and $k=(d)^{1 / \eta}=\log ^{c / \eta} d^{\prime}$. From observation (i) above, this truncation can be performed by a poly $\left(d^{\prime}\right)$-sized $A C^{0}$ circuit for each of the $d$ coefficients.

In order to deal with the iterated multiplication of $\mathbf{a} \in R_{q}$ with the secrets $\mathbf{s}_{1}, \ldots, \mathbf{s}_{k} \in R_{q}$, as before we first convert the ring elements from their standard representation to the canonical embedding $\sigma: R_{q} \rightarrow \mathbb{C}^{d}$ so that every element $z \in R_{q}$ is a complex vector of length $d=O\left(\right.$ polylog $\left.d^{\prime}\right)$ and each vector entry of size at most $O(\log q)=O\left(\right.$ polylog $\left.d^{\prime}\right)$ bits [LPR13b]. The complexity of the FFT and CRR operations needed to convert to and from the canonical embedding $\sigma$ in this case are constrained by the complexity of multiplication and exponentiation of $O(\log q)$-bit numbers. Then, using a variant of observation (ii) above that also works for exponentiation [HAB02, HV06], these operations can be performed by poly $\left(2^{(\log q)^{\varepsilon}}, 2^{k^{\varepsilon}}\right)$-sized $\mathrm{AC}^{0}$ circuits of depth $O(1 / \varepsilon)$. Finally, for the $d$-parallel iterated multiplications of at most $k+1$ elements each of $O(\log q)$-bits, by observation (ii) we need poly $\left(2^{(\log q)^{\varepsilon}}, 2^{k^{\varepsilon}}\right)$-sized $\mathrm{AC}^{0}$ circuits of depth $O(1 / \varepsilon)$ to evaluate it.

The total circuit size for evaluating $f_{\mathbf{a}, \mathbf{s}_{1}, \ldots, \mathbf{s}_{k}}(x)$ hinges on the complexity of $\operatorname{poly}\left(2^{(\log q)^{\varepsilon}}, 2^{k^{\varepsilon}}\right)$ which we show to be $O\left(\right.$ poly $\left.\left(d^{\prime}\right)\right)$ below. First, notice that $2^{k^{\varepsilon}} \leq 2^{2^{\left(\frac{k}{2} \log d\right)^{\varepsilon}}} \leq 2^{\left(\frac{k^{2}}{k+1} \log d\right)^{\varepsilon}}=2^{(\log q)^{\varepsilon}}$ for

\footnotetext{
${ }^{16}$ Basic arithmetic operations can be expressed by circuits containing AND, NOT, OR and MAJ gates. MAJ gates with $O($ polylog $n)$-bit fan-in can be computed by $\mathrm{AC}^{0}$ circuits.
} 
$d>4$. Hence, it suffices to bound only the complexity of the latter term in terms of $d^{\prime}$ :

$$
\begin{array}{rlr}
(\log q)^{\varepsilon} & =\left(\frac{k^{2}}{k+1} \log d\right)^{\varepsilon} \leq(k \log d)^{\varepsilon}=(d)^{\frac{\varepsilon}{\eta}}(\log d)^{\varepsilon} & \left(\text { Since, } k=(d)^{\frac{1}{\eta}}\right) \\
& =\left(\log d^{\prime}\right)^{\frac{c \varepsilon}{\eta}} O\left(\left(\log \log d^{\prime}\right)^{\varepsilon}\right) & \\
& \leq\left(\log d^{\prime}\right)^{\frac{c \varepsilon}{\eta}} O\left(\left(\log d^{\prime}\right)^{\varepsilon}\right) & \\
& =O\left(\left(\log d^{\prime}\right)^{\left(\frac{c}{\eta}+1\right) \varepsilon}\right) & \\
& =O\left(\left(\log d^{\prime}\right)^{\frac{c+\eta}{\eta} \varepsilon}\right) & \\
& =O\left(\log d^{\prime}\right) & \text { (Since, } \left.\varepsilon \leq \frac{\eta}{\eta+c}\right),
\end{array}
$$

which implies that $2^{(\log q)^{\varepsilon}} \leq 2^{O\left(\log d^{\prime}\right)}=O\left(\operatorname{poly}\left(d^{\prime}\right)\right)$.

Putting all the steps together, the function $f_{\mathbf{a}, s_{1}, \ldots, s_{k}}(x)$ taking as input a $k$-bit number $x$ can be computed by an $A C^{0}$ circuit of size $O\left(\operatorname{poly}\left(d^{\prime}\right)\right)=O\left(\operatorname{poly}\left(2^{1^{1 / c}}\right)\right)=2^{O\left(d^{1 / c}\right)}$ and depth scaling as $O(1 / \varepsilon)$.

\section{Quantum-secure PRFs vs. quantum learning}

In this section we prove our main theorem which shows the connection between efficient quantum learning and quantum algorithms which serve as distinguishers for pseudo-random functions. The structure of our proof goes in the same lines of the work of Kharitonov [Kha93, Kha92]: we assume the existence of an efficient learner for some PRF $\mathcal{F}$ and utilize it to construct an efficient distinguisher for $\mathcal{F}$ from truly random functions. However, we need here very different techniques, since the arguments used by Kharitonov do not follow in the quantum setting.

A key part of the proof for this theorem hinges on bounding how well a quantum algorithm predicts the output of a random function on a random input. We assume that the algorithm is allowed to query an oracle to the function (in superposition) at most q times. Each of these queries denotes performing a membership query to $z$, the truth-table of the function. We show an upper bound on the probability of predicting the output of this random function for a uniformly random input.

Lemma 4.1 Let $k>1$ and $f:\{0,1\}^{k} \rightarrow\{0,1\}$ be a random function. Consider a quantum algorithm that makes q quantum membership queries to $f$. Given a uniformly random question $x \in\{0,1\}^{k}$, the probability of the quantum algorithm correctly predicts $f(x)$ is

$$
\operatorname{Pr}_{x \in\{0,1\}^{k}}\left[h_{x}=f(x)\right] \leq \frac{1}{2}+\sqrt{\frac{k \cdot q}{2^{k}}},
$$

where $h_{x}$ is the output of the algorithm given the question $x$.

Proof. Let us denote $\gamma(k)=\sqrt{\frac{k \cdot q}{2^{k}}}$. On input some index $x$, we view $h_{x}$ as the output of a "hypothesis function" $h:\{0,1\}^{k} \rightarrow\{0,1\}$, such that $h_{x}=h(x)$. By contradiction, let us assume that the hypothesis function $h$ satisfies

$$
\operatorname{Pr}_{x \in\{0,1\}^{k}}[f(x)=h(x)]>\frac{1}{2}+\frac{\gamma(k)}{2},
$$


where $x$ is drawn uniformly from $\{0,1\}^{k}$. In this case, the mutual information between the truth table of $f$, which we denote as $z$, and the truth-table of the hypothesis $h$ is given by

$$
\mathbf{I}(z: h)=\sum_{x \in\{0,1\}^{k}} \mathbf{I}\left(z_{x}: h\right)=\sum_{x \in\{0,1\}^{k}}\left(\mathbf{H}\left(z_{x}\right)-\mathbf{H}\left(z_{x} \mid h\right)\right)=2^{k}-\sum_{x \in\{0,1\}^{k}} \mathbf{H}\left(z_{x} \mid h\right),
$$

where the first and last equality used the independence of the $z_{\chi}$ s because the truth table of $f$, i.e., $z \in\{0,1\}^{2^{k}}$, is a uniformly random string. By Fano's inequality (in Lemma 2.2), it follows that $\mathbf{H}\left(z_{x} \mid h\right) \leq \mathbf{H}_{b}\left(p_{x}\right)$, where $\mathbf{H}_{b}(\cdot)$ is the binary entropy function and $p_{x}$ is the probability of error on guessing $z_{x}$ by an arbitrary estimator whose input is $h:\{0,1\}^{k} \rightarrow\{0,1\}$. By assumption of $h$ in Equation (4), it follows that $\frac{1}{2^{k}} \sum_{x} p_{x}<\frac{1}{2}-\frac{\gamma(k)}{2}$. Using this, we then have that

$$
\sum_{x} \mathbf{H}\left(z_{x} \mid h\right) \leq \max _{\left\{p_{x}\right\}} \sum_{x} \mathbf{H}_{b}\left(p_{x}\right),
$$

where the maximization is over $\left\{p_{x}\right\}$ in the set $\left\{p_{x}: \frac{1}{2^{k}} \sum_{x} p_{x} \leq \frac{1}{2}-\frac{\gamma(k)}{2}\right\}$. Since $\mathbf{H}_{b}$ is a concave function, the maximum is obtained when all the $p_{x}$ s are equal (this also follows from Jensen's inequality). Given our upper-bound on the sum $\sum_{x} p_{x}$, it follows that $\sum_{x} \mathbf{H}_{b}\left(p_{x}\right)$ is maximized when $p_{x}=\frac{1}{2}-\frac{\gamma(k)}{2}$ for every $x$. In this case, we have

$$
\max _{\left\{p_{x}\right\}} \sum_{x} \mathbf{H}_{b}\left(p_{x}\right) \leq \sum_{x} \mathbf{H}_{b}\left(\frac{1}{2}-\frac{\gamma(k)}{2}\right)=2^{k} \cdot \mathbf{H}_{b}\left(\frac{1}{2}-\frac{\gamma(k)}{2}\right) \leq 2^{k}\left(1-\frac{2 \gamma(k)^{2}}{\ln 2}\right),
$$

where we use Fact 2.1 in the last inequality. Putting together Equation (5) and (6), we obtain

$$
\mathbf{I}(z: h)>\frac{2^{k+1} \gamma(k)^{2}}{\ln 2}>2 \cdot k \cdot \mathrm{q} .
$$

We now upper bound the mutual information between the output hypothesis $h$ and the uniformly random string $z$. One way to view the quantum algorithm is as a protocol where a quantum membership query to $z$ is a message from the algorithm to an oracle hiding $z$ and the oracle's output is a message from the oracle to the algorithm. In this case, using Corollary 2.3 the mutual information between the output hypothesis $h$ and the random string $z$ can be upper-bounded by the communication complexity of the protocol,

$$
\mathbf{I}(h: z) \leq(k+1) \cdot \mathrm{q} \text {. }
$$

However, since $k \geq 2$, the lower bound in Equation (7) contradicts Equation (8), which in turn contradicts our assumption in Equation (4).

Now, we can prove the main technical result of this section.

Theorem 4.2 Let $n, D \geq 2$ and $k=D \cdot \log n$. Let $\mathcal{F}=\left\{f_{K}:\{0,1\}^{k} \rightarrow\{0,1\} \mid K \in\{0,1\}^{n}\right\}$ be a family of functions. Suppose there exists a $t(n)$-time uniform quantum-PAC learner for $\mathcal{F}$ (given access to $\mathrm{q}$ quantum membership queries and uniform quantum examples) with bias $\beta(n) \geq 2 \sqrt{\frac{k \cdot q}{n^{D}}}$

Then there exists an $O(t(n))$-time quantum algorithm $\mathcal{D}$ that satisfies:

$$
\left|\operatorname{Pr}_{f \sim \mathcal{F}}\left[\mathcal{D}^{|f\rangle}(\cdot)=1\right]-\operatorname{Pr}_{f \sim \mathcal{U}}\left[\mathcal{D}^{|f\rangle}(\cdot)=1\right]\right| \geq \frac{\beta(n)}{2},
$$

where the probability is taken uniformly over $f \in \mathcal{F}$ and $f \in \mathcal{U}$, where $\mathcal{U}$ is the set of all functions from $\{0,1\}^{k}$ to $\{0,1\}$.

Proof. Let $\mathcal{A}$ be a $t(n)$-time uniform quantum-PAC learner that makes at most q quantum queries to a concept $c \in \mathcal{C}$ and outputs a hypothesis $h:\{0,1\}^{k} \rightarrow\{0,1\}$ such that

$$
\operatorname{Pr}_{x \in\{0,1\}^{k}}[c(x)=h(x)] \geq \frac{1}{2}+\beta(n),
$$

where the probability is over $x$ drawn uniformly from $\{0,1\}^{k}$. Note that $\mathcal{A}$ can obtain a uniform 
quantum example by making a single quantum membership query, so without loss of generality we assume $\mathcal{A}$ makes membership queries.

The goal is to use $\mathcal{A}$ to construct a quantum distinguisher $\mathcal{D}$ for the PRF $\mathcal{F}$ that satisfies Equation (9). Let the distinguisher $\mathcal{D}$ have quantum oracle access to the function $f:\{0,1\}^{k} \rightarrow\{0,1\}$. The goal for $\mathcal{D}$ is to decide if $f$ is a uniformly random function or if $f \in \mathcal{F}$. In order to do this, $\mathcal{D}$ proceeds by first running the quantum learning algorithm $\mathcal{A}$ as follows: whenever $\mathcal{A}$ makes a quantum membership query, $\mathcal{D}$ uses its oracle to answer $\mathcal{A}$.

After making q membership queries, $\mathcal{A}$ then outputs a hypothesis $h$. The distinguisher $\mathcal{D}$ outputs 1 (i.e., $f$ is a pseudo-random function) if and only if $h(x)=f(x)$ for a uniformly random $x \in\{0,1\}^{k}$.

The running time of $\mathcal{D}$ is $O(t(n))$ since $\mathcal{A}$ runs in time $t(n)$, each query has constant cost and computing $h(x)$ takes at most $O(t(n))$ time. We now show that $\mathcal{D}$ satisfies Equation (9). Suppose $f$ is a pseudo-random function i.e., $f \in \mathcal{F}$. Each query from $\mathcal{A}$ will be answered correctly as $\mathcal{D}$ has oracle access to it. Hence, we have

$$
\operatorname{Pr}\left[\mathcal{D}^{|f\rangle}(\cdot)=1\right]=\operatorname{Pr}_{x \in\{0,1\}^{k}}[f(x)=h(x)] \geq \frac{1}{2}+\beta(n),
$$

where the inequality follows from the correctness of the quantum learning algorithm $\mathcal{A}$.

On the other hand, if $f$ was a uniformly random function, using Lemma 4.1, we can conclude that

$$
\operatorname{Pr}\left[\mathcal{D}^{|f\rangle}(\cdot)=1\right]=\operatorname{Pr}_{x \in\{0,1\}^{k}}[f(x)=h(x)] \leq \frac{1}{2}+\sqrt{\frac{k \cdot \mathrm{q}}{2^{k}}}=\frac{1}{2}+\sqrt{\frac{k \cdot \mathrm{q}}{n^{D}}}
$$

where the last equality uses the definition of $k=D \cdot \log n$.

Combining these two cases, the bias of the distinguisher $\mathcal{D}$ is

$$
\left|\operatorname{Pr}_{f \in \mathcal{F}}\left[\mathcal{D}^{|f\rangle}(\cdot)=1\right]-\operatorname{Pr}_{f \in \mathcal{U}}\left[\mathcal{D}^{|f\rangle}(\cdot)=1\right]\right| \geq\left(\frac{1}{2}+\beta(n)\right)-\left(\frac{1}{2}+\sqrt{\frac{k \cdot \mathrm{q}}{n^{D}}}\right)=\beta(n)-\sqrt{\frac{k \cdot \mathrm{q}}{n^{D}}} \geq \frac{\beta(n)}{2},
$$

where the last inequality uses the definition of $\beta(n)$. This concludes the proof.

Our hardness results for $\mathrm{TC}^{0}$ and $\mathrm{AC}^{0}$ are based of the following corollary. In order for notational simplicity in the subsequent sections, we replace $n$ in Theorem 4.2 by $M$ below.

Corollary 4.3 Let $M \geq 2, D \in\{2, \ldots, M /(4 \log M)\}$ and $a>0$ be a constant. Let $\mathcal{F}=\left\{f_{K}:\{0,1\}^{k} \rightarrow\right.$ $\left.\{0,1\} \mid K \in\{0,1\}^{M}\right\}$ be a family of functions for some $k=D \cdot \log M$.

For any $t(M) \leq M^{D-a}$ and $\beta(M) \geq 2 \sqrt{\frac{k}{M^{a}}}$, if there exists a $t(M)$-time uniform quantum-PAC learner for $\mathcal{F}$ with quantum membership queries and bias $\beta(M)$, then there exists a distinguisher that runs in time $O(t(M))$ and is able to distinguish $\mathcal{F}$ from a uniform random function.

Proof. First observe that the number of queries made by the learner is at most $\mathrm{q} \leq M^{D-a}$. Hence,

$$
\beta(M) \geq 2 \sqrt{\frac{k}{M^{a}}}=2 \sqrt{\frac{k M^{D-a}}{M^{D}}} \geq 2 \sqrt{\frac{k \cdot \mathrm{q}}{M^{D}}},
$$

which satisfies the assumption of Theorem 4.2. Hence, there is a $O(t(M))$-time quantum distinguisher for the PRF family $\mathcal{F}$ with distinguishing probability $\beta(M)=1 / \operatorname{poly}(M)$ since we let $a>0$ be a constant. 


\subsection{Hardness of learning $\mathrm{TC}^{0}$}

For completeness, we formally state Result 3 and follow with its proof.

Theorem 4.4 Let $n \in \mathbb{N}, q=2^{O\left(n^{c}\right)}$ for some constant $c \in(0,1), r \leq \operatorname{poly}(n)$ and $t(n) \leq 2^{O(\operatorname{polylog}(n))}$. Let $\Upsilon(r)$ be the n-dimensional discrete Gaussian distribution over a degree-n cyclotomic ring with standard deviation at most $r$ in each coefficient. Assuming that the $\operatorname{RLWE}_{n, q, \Upsilon(r)}$ problem is hard for an $O(t(n))$ time quantum computer, then there is no $O(t(n))$-time uniform weak quantum-PAC learner for poly $(n)$ sized $\mathrm{TC}^{0}$ circuits.

Proof. We proceed by a proof of contradiction. First, consider the concept class to be the $1 \mathrm{~B}-\mathrm{RPRF}_{n, q, p, k}$ function family $\mathcal{R} \mathcal{F}$ as given by Definition 3.13, where $2 \leq p \ll q$ are powers of 2 , $k=\log ^{\alpha} n=\omega(\log n)$ for some constant $\alpha>2, p=2$ and $q=2^{\gamma \cdot \frac{k}{k+1}}$ and $\gamma=n^{c}$ for $0<c<1$. Using Lemma 3.16 for $d=n$, every function in $\mathcal{R} \mathcal{F}$ can be computed by an $O(\operatorname{poly}(n), \omega(\log n))=$ $O($ poly $(n))$-sized $\mathrm{TC}^{0}$ circuit. In other words, the concept class $\mathcal{R} \mathcal{F} \subseteq \mathrm{TC}^{0}$.

By way of contradiction, suppose that there is a $t(n)$-time uniform weak quantum learning algorithm for $\mathcal{R} \mathcal{F}$, i.e., there exists a learner $\mathcal{A}$ for $\mathcal{R} \mathcal{F}$ that uses at most $t(n)$ queries/samples and achieves bias $\beta(n)=n^{-\delta}$ for some constant $\delta>0$.

Let $M=(k+1) \log q=\gamma k=\gamma \log ^{\alpha} n$ (where the first equality used that $\log q=\gamma \cdot k /(k+1)$ by definition) and $D=\frac{k}{\log M}=\frac{\log ^{\alpha} n}{\log \gamma+\alpha \log \log n}$. Let $a$ be some constant satisfying $a \geq 2(\delta+1) / c$. This choice of $a$ allows us to show that

$$
\beta(n) \geq \frac{1}{n^{\delta}} \geq \frac{1}{n^{c a / 2-1}} \geq 2 \sqrt{\frac{(\log n)^{\alpha(1-a)}}{n^{c a}}}=2 \sqrt{\frac{(\log n)^{\alpha(1-a)}}{\gamma^{a}}}=2 \sqrt{\frac{\log ^{\alpha} n}{\left(\gamma \log ^{\alpha} n\right)^{a}}}=2 \sqrt{\frac{k}{M^{a}}},
$$

where the last equality uses the definition of $M$ and the penultimate equality uses the definition of $\gamma$. Finally, notice that

$$
D-a=\frac{\log ^{\alpha} n}{\log \gamma+\alpha \log \log n}-a=\frac{\log ^{\alpha} n}{c \log n+\alpha \log \log n}-a \geq \frac{\log ^{\alpha-1} n}{2 c},
$$

using $\gamma=n^{c}$ in the second equality. Therefore we have

$$
M^{D-a} \geq\left(\gamma \log ^{\alpha} n\right)^{\frac{\log ^{\alpha-1} n}{2 c}}=2^{O(\operatorname{polylog}(n))} \geq t(n) .
$$

This choice of parameters allows us to use Corollary 4.3, which implies the existence of an $O(t(n))$ time distinguisher for the $1 \mathrm{~B}-\mathrm{RPRF}_{n, q, p, k}$ family $\mathcal{R} \mathcal{F}$. For the aforementioned choices of $n, q$ and $r$, using Lemma 3.15, we can translate the algorithm for $\mathcal{R} \mathcal{F}$ to an $O(t(n))$-time quantum distinguisher for the $\mathrm{RLWE}_{n, q, \Upsilon} \Upsilon$ problem, which contradicts our assumption.

An immediate corollary of Theorem 4.4 using Assumptions 1 and 2 gives us our main results:

Corollary 4.5 Let $n \in \mathbb{N}, q=2^{O\left(n^{c}\right)}$ for some constant $0<c<1, r \leq \operatorname{poly}(n)$ and $\Upsilon(r)$ be discrete Gaussian distribution over degree-n cyclotomic rings with standard deviation at most $r$ in each coefficient.

1. Assuming that the $\mathrm{RLWE}_{n, q, \Upsilon(r)}$ problem is hard for a poly $(n)$-time quantum computer, then there is no $\operatorname{poly}(n)$-time uniform weak quantum-PAC learner for $\operatorname{poly}(n)$-sized $\mathrm{TC}^{0}$ circuits.

2. Assuming that the $\operatorname{RLWE}_{n, q, \Upsilon(r)}$ problem is hard for a $2^{O(\operatorname{polylog}(n))}$-time quantum computer, then there is no $2^{O(\operatorname{polylog}(n))}$-time uniform weak quantum-PAC learner for poly $(n)$-sized $\mathrm{TC}^{0}$ circuits.

\subsection{Hardness of learning $A C^{0}$}

The proof for the hardness of learning $\mathrm{AC}^{0}$ is similar to the proof in the previous section and will proceed by showing that for a suitable choice of parameters, the concept class constructed 
from $\mathcal{R F}$ can be evaluated by $\mathrm{AC}^{0}$ circuits. Recall from Lemma 3.17, that the circuit size scales sub-exponentially with respect to the dimension of the underlying problem. Hence, in order to prove the $\mathrm{AC}^{0}$ hardness, we make two changes. We first consider "smaller instances" of the 1B-RPRF that can now be computed in $\mathrm{AC}^{0}$ (instead of $\mathrm{TC}^{0}$ as we showed in Lemma 3.16). Secondly, we "weaken" the statement of our hardness result by showing that, the existence of a quasi-polynomial time strong quantum learner for $\mathrm{AC}^{0}$ implies the existence of a strongly subexponential time quantum distinguisher for RLWE (instead of polynomial-time quantum distinguishers for RLWE as we showed in Theorem 4.4).

We first give reasons as to why the strongly sub-exponential time assumption is justified. The best known algorithms for $d$-dimensional LWE using various methods from lattice reduction techniques [LLL82, CN11], combinatorial techniques [BKW03, Wag02] or algebraic ones [AG11] all require $2^{O(d)}$-time in the asymptotic case. Even by quantizing any techniques, the improvements so far only affect the constants in the exponent and do not provide general sub-exponential time algorithms for LWE. It is also believed that the best-known algorithms for Ring-LWE do not do much better than the lattice reduction/combinatorial methods used for LWE. While the exponential run-times correspond to the hardest instances of these problems, for the choice of parameters used in this section, the algorithms scale more like $2^{\Omega(\sqrt{d})}$. Hence, we are justified in weakening our statement of hardness to the assumption that RLWE does not have $2^{d^{1 / \eta}}$-time algorithms for every constant $\eta>2$.

Before going into the proof, we provide some intuition as to why the choice of parameters as defined in Lemma 3.17 forces us to impose stronger hardness assumptions - the circuits are poly $(n)$-sized and the algorithms run in poly $(n)$-time but, the family $\mathcal{R} \mathcal{F}$ is defined on a ring of smaller dimension i.e., $d=O$ (polylog $n)$. However, from the perspective of the input size which scales as poly $(d)$, this means that the algorithms scale sub-exponentially in $d$. Hence, we need to use the more stringent "no strongly sub-exponential time algorithms for RLWE" assumption. We formally restate Result 5 below and proceed with its proof.

Theorem 4.6 Let $c>\eta>2$ such that $c / \eta>2$, and $a \geq 2$ be constants. Let $n \in \mathbb{N}, d=\log ^{c} n, k=d^{1 / \eta}$, $q=d^{O(k)}$ and $r \leq d^{\lambda}$ for $0<\lambda<1 / 2-O(1 / k), \Upsilon(r)$ be discrete Gaussian distribution over degree$n$ cyclotomic rings with standard deviation at most $r$ in each coefficient. Let $t(d) \leq 2^{d^{1 / \eta}}$ and $\beta(n) \geq$ $2(\log n)^{c(1-2 a) / 2 \eta}$. Assuming that $\operatorname{RLWE}_{d, q, \Upsilon(r)}$ is hard for a $t(d)$-time quantum computer, there is no $2^{(\log n)^{c / \eta}}$-time uniform quantum-PAC learner with bias $\beta(n)$ for $\operatorname{poly}(n)$-sized $\operatorname{AC}^{0}$ circuits.

Proof. Consider the constants $c, \eta, \varepsilon$ such that $c>\eta>2$ and $\varepsilon \leq \frac{\eta}{\eta+c}$. Consider the concept class $\mathcal{R F}$ from the function family $1 \mathrm{~B}-\mathrm{RPRF}_{d, q, p, k}$ as given by Definition 3.13 where dimension $d=$ $\log ^{c} n$, input $k=d^{\frac{1}{\eta}}$, and moduli $2<p \ll q$ are powers of 2 such that $\log q=\frac{k^{2} \log d}{k+1}$. By Lemma 3.17, every function in $\mathcal{R F}$ can be computed by a $2^{O\left(d^{1 / c}\right)}$-sized $A C^{0}$ circuit of depth $O(1 / \varepsilon)$ which is $O(1)$ since $\varepsilon \leq \eta /(\eta+c) \leq 1$. In other words, the $\mathrm{AC}^{0}$ circuits are of size $\left.2^{O\left((\log n)^{c \cdot 1 / c}\right.}\right)=O(\operatorname{poly}(n))$.

We now consider a function class $\mathcal{R} \mathcal{F}$ against which we are going to prove our hardness result: keeping Lemma 3.17 in mind, recall that the length of the key (i.e., number of bits to define the parameters $\mathbf{a}, \mathbf{s}_{i} \in R_{q}$ of the function $\left.f_{\mathbf{a}, \mathbf{s}_{1}, \ldots, \mathbf{s}_{k}}\right)$ is at most $M:=(k+1) \log q=d^{2 / \eta} \log d$, let

$$
\mathcal{R F}=\left\{f_{K}:\{0,1\}^{k} \rightarrow\{0,1\} \mid K \in\{0,1\}^{(k+1) \log q}\right\} .
$$

In order to discuss the hardness of learning the function class $\mathcal{R} \mathcal{F}$ in terms of the hardness of $d$ dimensional RLWE, we now relabel the function class above as follows: since $(k+1) \log q=k^{2} \log d$ and $k=d^{1 / \eta}$, we have:

$$
\mathcal{R} \mathcal{F}=\left\{f_{K}:\{0,1\}^{k} \rightarrow\{0,1\} \mid K \in\{0,1\}^{d^{2 / \eta} \log d}\right\} .
$$


By way of contradiction, suppose that there exists a $t(d) \leq 2^{d^{1 / \eta}}=2^{(\log n)^{c / \eta}}$-time uniform quantum learner, with bias $\beta(n)$, for poly $(n)$-sized $\mathrm{AC}^{0}$ circuits i.e., there exists a learner $\mathcal{A}$ for $\mathcal{R} \mathcal{F}$ that uses at most $t(d)$ queries/samples and achieves bias $\beta(n)$.

Let $D=\frac{\sqrt{M}}{\log M}$. Observe that $k=D \cdot \log M$,

$$
\beta(n) \geq 2(\log n)^{c(1-2 a) / 2 \eta}=2 d^{\frac{1}{2 \eta}-\frac{a}{\eta}}=2 \sqrt{\frac{d^{1 / \eta}}{d^{2 a / \eta}}} \geq 2 \sqrt{\frac{k}{M^{a}}} .
$$

Additionally, observe that

$$
(D-a) \log M=\left(\frac{\sqrt{M}}{\log M}-a\right) \cdot \log M \geq \sqrt{M} / 2 \geq d^{1 / \eta}(\sqrt{\log d}) / 2 \geq d^{1 / \eta}
$$

Hence, it follows that $M^{D-a} \geq 2^{d^{1 / \eta}} \geq t(d)$. We are now ready to apply Corollary 4.3 since $M, k, a$ and $D$ satisfy the required properties. It now follows from Corollary 4.3 that there exists an $O(t(d))$ time distinguisher for the $1 \mathrm{~B}-\mathrm{RPRF}_{d, q, p, k}$ problem with distinguishing advantage at least $\beta(n) / 2 \geq O(1 / \operatorname{poly}(d))$, from Equation (11). For the aforementioned choices of $d, q$ and $r$, using Lemma 3.15, this distinguisher can be converted into an $O(t(d))$ time algorithm for the $\operatorname{RLWE}_{d, q, \Upsilon(r)}$ problem with an $O(1 /$ poly $(d))$ distinguishing advantage. This contradicts the assumption that algorithms for $\operatorname{RLWE}_{d, q, \Upsilon(r)}$ require $2^{\Omega\left(d^{1 / \eta}\right)}$-time and concludes the proof.

While the previous theorem lower bounds shows that there is no quasi-polynomial-time algorithm to learn $\mathrm{AC}^{0}$ circuits with $\beta(n) \geq O(1 /$ polylog $n)$, we can weaken our assumption to also consider strong learners with bias $\beta(n) \geq O(1)$ (say, $\beta=1 / 6$ ). Setting $\beta(n)=1 / 6$ in Theorem 4.6 , we would obtain a distinguisher for $d$-dimensional RLWE that runs in time $2^{O\left(d^{1 / c}\right)}$ with a distinguishing advantage $\beta(n)=1 / 12 \geq 1 /$ poly $(d)$ thereby contradicting the sub-exponential time hardness of RLWE. Using Assumption 3, this leads to the following corollary which is the main result in this section.

Corollary 4.7 Let $c>\eta>2, n \in \mathbb{N}, d=\log ^{c} n, k=d^{1 / \eta}, q=d^{O(k)}$ and $r \leq d^{\lambda}$ for $0<\lambda<1 / 2-O(1 / k)$. Let $\Upsilon(r)$ be the discrete Gaussian distribution over a degree- $n$ cyclotomic ring with standard deviation at most $r$ in each coefficient. Assuming that the $\mathrm{RLWE}_{d, q, \Upsilon(r)}$ problem is hard for a $2^{o\left(d^{1 / \eta}\right)}$-time quantum

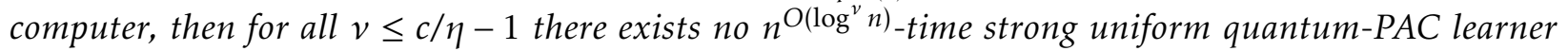
can learn $\operatorname{poly}(n)$-sized $\mathrm{AC}^{0}$ circuits.

Notice that this matches the $\Omega\left(n^{\text {polylog } n}\right)$ lower bound for classical learning AC $^{0}$ as obtained by Kharitonov [Kha93] under the assumption factoring is quasi-polynomial-hard. In particular, this lower bound also matches the $O\left(n^{\log ^{\alpha} n}\right)$ sample and time complexity upper bound for learning $\mathrm{AC}^{0}$ circuits as demonstrated by Linial, Mansour and Nisan [LMN93] for some universal constant $\alpha>1$. Hence, we can conclude that uniform strong quantum-PAC learners cannot offer a significant advantage for learning $A C^{0}$ circuits.

Using PRFs based on RLWR and our proof technique, we believe that it may not be possible to find a lower bounds for weak quantum learners for $\mathrm{AC}^{0}$ circuits. The reason is two-fold: (1) The circuit size of the PRF family scales as sub-exponential in the dimension $d$ while the key-length scales as $\operatorname{poly}(d)=O(\operatorname{polylog} n)$ for $\mathrm{AC}^{0}$ circuits and (2) this limits the bias $\beta(n)$ to be bounded from below by $O(1 / \operatorname{poly}(d))=O(1 /$ polylog $n)$ while weak learners are expected to have a bias $\beta(n) \geq O(1 / \operatorname{poly}(n))$. We leave the question of whether a different choice of quantum-secure PRFs with a more efficient circuit implementation could improve the lower bound open for future work. 


\section{Quantum-secure encryption vs. quantum learning}

In this section, we prove a theorem relating the security of quantum public-key cryptosystems and the hardness of quantum learning the class of decryption functions in the cryptosystem.

Kearns and Valiant [KV94] showed the simple, yet powerful connection between learning and public-key cryptography: consider a secure public-key cryptosystem and define a concept class $\mathcal{C}$ as the set of all decryption functions (one for every public and private key). Assume that there was an efficient PAC learner for $\mathcal{C}$. Given a set of encryptions (which should be viewed as labeled examples), supposing an efficient learner for $\mathcal{C}$ was able to learn an unknown decryption function with non-negligible advantage, then this learner would break the cryptosystem. This contradicts the assumption that the cryptosystem was secure and, in turn, shows the infeasibility of learning $\mathcal{C}$. Kearns and Valiant used this connection to give hardness results for learning polynomialsized formulas, deterministic finite automata, and constant-depth threshold circuits based on the assumption that Blum integers are hard to factor or that it is hard to invert the RSA function. Our main contribution in this section is to quantize the theorem by Kearns and Valiant [KV94] and draw a relation between quantum learning and security of quantum cryptosystems.

Theorem 5.1 Consider a public-key cryptosystem which encrypts bits by n-bit strings. Suppose that the (randomized) encryption function is given by $e_{\mathrm{K}_{\mathrm{pub}}, r}:\{0,1\} \rightarrow\{0,1\}^{n}$ (where $K=\left(\mathrm{K}_{\text {pub }}, \mathrm{K}_{\mathrm{priv}}\right.$ ) consists of the public and private keys and $r$ is a random string) and the decryption function is given by $d_{\mathrm{K}_{\text {priv }}}$ : $\{0,1\}^{n} \rightarrow\{0,1\}$. Let $\mathcal{C}=\left\{d_{\mathrm{K}_{\text {priv }}}:\{0,1\}^{n} \rightarrow\{0,1\}\right\}_{\mathrm{K}_{\text {priv }}}$ be a concept class. Let

$$
\varepsilon=\underset{K, r}{\operatorname{Pr}}\left[d_{\mathrm{K}_{\text {priv }}}\left(e_{\mathrm{K}_{\mathrm{pub}}, r}(0)\right) \neq 0 \text { or } d_{\mathrm{K}_{\mathrm{priv}}}\left(e_{\mathrm{K}_{\mathrm{pub}}, r}(1)\right) \neq 1\right] \text {, }
$$

be the negligible probability of decryption error (where the probability is taken over uniformly random $K=\left(\mathrm{K}_{\text {pub }}, \mathrm{K}_{\text {priv }}\right)$ and $\left.r\right)$. If there exists a weak quantum-PAC learner for $\mathcal{C}$ in time $t(n) \geq n$ that satisfies $t(n) \beta(n)=1 / n^{\omega(1)}$, then there exists a $t(n)$-time quantum algorithm Adv that satisfies

$$
\left|\operatorname{Pr}_{K, r^{*}, b^{*}}\left[\operatorname{Adv}\left(\mathrm{K}_{\mathrm{pub}}, e_{\mathrm{K}_{\mathrm{pub}}, r^{*}}\left(b^{*}\right)\right)=b^{*}\right]-\operatorname{Pr}_{K, r^{*}, b^{*}}\left[\operatorname{Adv}\left(\mathrm{K}_{\mathrm{pub}}, e_{\mathrm{K}_{\mathrm{pub}}, r^{*}}\left(b^{*}\right)\right)=\overline{b^{*}}\right]\right| \geq \frac{1}{\operatorname{poly}(n)},
$$

where the probability is taken over uniformly random $K, r^{*}, b^{*}$ over their respective domains.

Proof. For notational simplicity, for a fixed $\mathrm{K}_{\mathrm{pub}}$, let $e_{r, b}:=\operatorname{Enc}_{\mathrm{K}_{\mathrm{pub}}, r}(b)$ and $\mathcal{E}=\left\{e_{r, b}: r, b\right\}$ be the set of all encryptions for the public key $\mathrm{K}_{\text {pub }}$. Our goal here is to devise a quantum algorithm Adv that satisfies the following: on input $\left(\mathrm{K}_{\mathrm{pub}}, e^{*}\right)$, where $e^{*}=e_{r^{*}, b^{*}}$ is uniformly randomly chosen from $\mathcal{E}$, Adv outputs $\hat{b}$ such that $\hat{b}=b^{*}$ with $1 / \operatorname{poly}(n)$ advantage over a random guess. Adv would clearly serve as our quantum algorithm that satisfies Equation (12), thereby proving the theorem.

Our approach is to devise an Adv so that the remainder of our reasoning resembles the (classical) proof of this theorem by Klivans and Sherstov [KS09] (who in turn re-derived the proof of Kearns and Valiant [KV94] in their paper). However the quantization of their proof is not straightforward. Classically, it is possible to generate samples from the uniform distribution supported on $\mathcal{E}$ as follows: pick $r$ and $b$ uniformly at random from their respective domains and then output the uniform classical examples $\left(e_{r, b}, b\right)$. Quantumly, it is not clear how a polynomial-time adversary Adv could create a quantum example

$$
\frac{1}{\sqrt{|\mathcal{E}|}} \sum_{r, b}\left|e_{r, b}\right\rangle|b\rangle
$$

Notice that if an adversary could prepare the state in Equation (13), then it could directly pass $t(n)$ quantum examples to the quantum-PAC learner (assumed in the theorem statement) and a similar analysis as in [KS09] gives a quantum algorithm (or distinguisher) that satisfies Equation (12). However, one issue while preparing the state in Equation (13) is the following: the straightforward 
way to prepare the state Equation (13) would be create the uniform superposition $\frac{1}{\sqrt{|\mathcal{E}|}}|r\rangle|b\rangle|0\rangle$ and then in superposition prepare $\frac{1}{\sqrt{|\mathcal{E}|}}|r\rangle|b\rangle\left|e_{r, b}\right\rangle$. If there was a way to erase the first register, then we would get the state in Equation (13). However, erasing this register in a black-box way is equivalent to solving the index-erasure problem [AMRR11, LR19], which needs exponential time.

In order to circumvent the issue mentioned above, we now show how to tweak the proof and construct an adversary $A d v_{\text {real }}$ that correctly decrypt a challenge encryption (i.e., $e_{r^{*}, b^{*}}$ ) with nonnegligible probability. Before constructing $A d v_{\text {real }}$, we first present an adversary $A d v_{\text {ideal }}$ that is able to create specific samples (see Equation (14)) that $A d v_{\text {real }}$ could not create and analyze its success probability. Then we show that the success probability of $A d v_{\text {real }}$ and $A d v_{\text {ideal }}$ in predicting the challenge encryption (i.e., $e_{r^{*}, b^{*}}$ ) are very close.

Let $S_{\text {ideal }}=\left\{\left(r_{i}, b_{i}\right)_{i}\right\}$ be a fixed set of size $L=2 n^{c} t(n)$ that contains $\left(r^{*}, b^{*}\right)$ and let us assume $A d v_{\text {ideal }}$ has access to $S_{\text {ideal }}$. Then $A d v_{\text {ideal }}$ can create the quantum example

$$
\left|\psi_{\text {ideal }}\right\rangle=\frac{1}{\sqrt{L}} \sum_{(r, b) \in S_{\text {ideal }}}\left|e_{r, b}, b\right\rangle
$$

in polynomial time. Notice that since $A_{d v} v_{\text {real }}$ does not know the decryption of $e_{r^{*}, b^{*}}$, so it would most likely not be able to obtain such an $S_{\text {ideal }}$ and consequently would not be able to create $\left|\psi_{\text {ideal }}\right\rangle$ efficiently. We now continue our analysis for $A d v_{\text {ideal }}$ assuming it has access to quantum examples $\left|\psi_{\text {ideal }}\right\rangle . A d v_{\text {ideal }}$ first passes $t(n)$ copies of $\left|\psi_{\text {ideal }}\right\rangle$ to the quantum-PAC learning algorithm for $\mathcal{C}$ (which is assumed by the theorem statement). Let $\mathcal{H}(\mathcal{D})$ be the distribution of the hypothesis output by the quantum-PAC learning algorithm when the samples come from the distribution $\mathcal{D}$. For a set $S=\left\{\left(r_{i}, b_{i}\right)\right\}_{i}$, let $\mathcal{D}_{S}$ be the uniform distribution on the training set $\left\{\left(e_{r, b}, b\right):(r, b) \in S\right\}$. Suppose the PAC learning algorithm outputs a hypothesis $h \in \operatorname{supp}\left(\mathcal{H}\left(\mathcal{D}_{S}\right)\right)$, then $\mathcal{A}_{\text {ideal }}$ outputs $h\left(e_{r^{*}, b^{*}}\right)$ as its guess for $b^{*}$. We now analyze the probability that output of $\operatorname{Adv}_{\text {ideal }}$ is in fact the correct decryption of $e^{*}$ :

$$
\begin{aligned}
\operatorname{Pr}_{e^{*} \in U_{E}}\left[\operatorname{Adv}_{\text {ideal }}\left(e^{*}\right)=b^{*}\right] & =\operatorname{Pr}_{e^{*} \in U_{E}} \mathbb{E}_{S \subseteq E}\left[\operatorname{Adv}_{\text {ideal }}\left(e^{*}\right)=b^{*}\left|e^{*} \in S \wedge\right| S \mid=L\right] \\
& =\operatorname{Pr}_{e^{*} \in U_{E}} \mathbb{E}_{S \subseteq E}\left[\underset{h \sim \mathcal{H}\left(\mathcal{D}_{S}\right)}{\operatorname{Pr}}\left[h\left(e^{*}\right)=b^{*}\right]\left|e^{*} \in S \wedge\right| S \mid=L\right] \\
& =\operatorname{Pr}_{e^{*} \in U_{E}} \sum_{S \subseteq E} \operatorname{Pr}_{\mathbf{S} \subseteq E}\left[\mathbf{S}=S\left|e^{*} \in S \wedge\right| S \mid=L\right] \underset{h \sim \mathcal{H}\left(\mathcal{D}_{S}\right)}{\operatorname{Pr}}\left[h\left(e^{*}\right)=b^{*}\right] \\
& =\sum_{S \subseteq E} \sum_{e^{*} \in S} \operatorname{Pr}_{\mathbf{S} \subseteq E}[\mathbf{S}=S|| S \mid=L] \operatorname{Pr}_{W \in U_{S}}\left[W=e^{*}\right] \operatorname{Pr}_{h \sim \mathcal{H}\left(\mathcal{D}_{S}\right)}\left[h\left(e^{*}\right)=b^{*}\right] \\
& =\sum_{S \subseteq E} \operatorname{Pr}_{\mathbf{S} \subseteq E}[\mathbf{S}=S \| S \mid=L] \underset{h \sim \mathcal{H}\left(\mathcal{D}_{S}\right)}{\operatorname{Pr}}\left[\mathbb{E}_{e^{*} \in S}\left[h\left(e^{*}\right)=b^{*}\right]\right] \\
& \geq \sum_{S \subseteq E} \operatorname{Pr}_{\mathbf{S} \subseteq E}[\mathbf{S}=S \| S \mid=L]\left(\frac{1}{2}+\frac{1}{n^{c}}\right)=\frac{1}{2}+\frac{1}{n^{c}} .
\end{aligned}
$$

In the first equality, the expectation is taken over uniformly random $S \subseteq E$ conditioned on $|S|=L$ and $e^{*} \in S$, second equality describes the operations of $A d v_{\text {ideal }}$, the fourth equality holds because

$$
\operatorname{Pr}_{e^{*} \in U_{E}} \sum_{S \subseteq E} \operatorname{Pr}_{\mathbf{S} \subseteq E}\left[\mathbf{S}=S\left|e^{*} \in S \wedge\right| S \mid=L\right]=\frac{1}{|E|}\left(\begin{array}{c}
E-1 \\
L-1
\end{array}\right)^{-1}=\left(\begin{array}{l}
E \\
L
\end{array}\right)^{-1} \frac{1}{L}=\sum_{S \subseteq E} \sum_{e^{*} \in S} \operatorname{Pr}_{\mathbf{S} \subseteq E}[\mathbf{S}=S \| S \mid=L] \operatorname{Pr}_{W \in U_{S}}\left[W=e^{*}\right]
$$


and the inequality comes from the guarantees of the quantum learning algorithm. ${ }^{17}$ Hence Equation (15) shows that the ideal quantum algorithm $A_{d v}$ ideal satisfies the following: on input a uniformly random $e^{*}=e_{r^{*}, b^{*}}$ from the set $E, \operatorname{Adv}_{\text {ideal }}$ outputs $b^{*}$ with probability at least $1 / 2+n^{-c}$.

We now consider Adv real. Let

$$
\left|\psi_{\text {real }}\right\rangle=\frac{1}{\sqrt{L-1}} \sum_{(r, b) \in S_{\text {real }}}\left|e_{r, b}, b\right\rangle,
$$

where $S_{\text {real }}=S_{\text {ideal }} \backslash\left\{e^{*}\right\}$. The idea is that for $L=2 n^{c} t(n)$, the distance between the two states $\left|\psi_{\text {ideal }}\right\rangle$ and $\left|\psi_{\text {real }}\right\rangle$ is small. Also notice that the state in Equation (16) can be constructed efficiently by $\operatorname{Adv}_{\text {real }}$ : pick $L-1$ many $\left(r_{i}, b_{i}\right)$-pairs uniformly at random from their respective domains and set $S_{\text {real }}=\left\{\left(r_{i}, b_{i}\right)_{i}\right\}$. From these classical values, the learner can create $t(n)$ many copies of the state $\left|\psi_{\text {real }}\right\rangle$. We now show that a hypothesis sampled from $\mathcal{H}\left(\mathcal{D}_{S_{\text {real }}}\right)$ behaves "almost similar" to a hypothesis sampled from $\mathcal{H}\left(\mathcal{D}_{S_{\text {ideal }}}\right)$. For every $y \in\{0,1\}^{n}$ and $b \in\{0,1\}$, observe that

$$
\begin{aligned}
\operatorname{Pr}_{h \sim \mathcal{H}\left(\mathcal{D}_{S_{\text {real }}}\right)}[h(y)=b] & =\operatorname{Pr}_{h \sim \mathcal{H}\left(\mathcal{D}_{S_{\text {real }}}\right)}[h(y)=b]-\operatorname{Pr}_{h \sim \mathcal{H}\left(\mathcal{D}_{S_{\text {ideal }}}\right)}[h(y)=b]+\operatorname{Pr}_{h \sim \mathcal{H}\left(\mathcal{D}_{S_{\text {ideal }}}\right)}[h(y)=b] \\
& \geq \operatorname{Pr}_{h \sim \mathcal{H}\left(\mathcal{D}_{S_{\text {ideal }}}\right)}[h(y)=b]-O\left(\frac{t(n)}{L}\right) .
\end{aligned}
$$

The inequality holds since the statistical distance of the output of an algorithm $A$ in inputs $|\phi\rangle$ and $\left|\phi^{\prime}\right\rangle$ is upper-bounded by $\||\phi\rangle-\left|\phi^{\prime}\right\rangle \|^{2}$, and we have that

$$
\|\left|\psi_{\text {ideal }}\right\rangle^{\otimes t(n)}-\left|\psi_{\text {real }}\right\rangle^{\otimes t(n)}\left\|^{2}=t(n)\right\|\left|\psi_{\text {ideal }}\right\rangle-\left|\psi_{\text {real }}\right\rangle \|^{2}=O\left(\frac{t(n)}{L}\right)
$$

by the definition of $\left|\psi_{\text {ideal }}\right\rangle$ and $\left|\psi_{\text {real }}\right\rangle$ in Equation (14) and 16 respectively. We now analyze the probability that $A d v_{\text {real }}$ outputs the right decryption $b^{*}$, when given a uniformly random sample $e^{*} \in E:$

$$
\begin{aligned}
& \operatorname{Pr}_{e^{*} \in U_{E}}\left[\operatorname{Adv}_{\text {real }}\left(e^{*}\right)=b^{*}\right]=\operatorname{Pr}_{e^{*} \in U_{E}} \mathbb{E}_{S_{\text {real }} \subseteq E}\left[\operatorname{Adv}_{\text {real }}\left(e^{*}\right)=b^{*}|| S_{\text {real }} \mid=L-1\right] \\
& =\operatorname{Pr}_{e^{*} \in U_{E}} \mathbb{E}_{S_{\text {real }} \subseteq E}\left[\operatorname{Pr}_{h \sim \mathcal{H}\left(\mathcal{D}_{S_{\text {real }}}\right)}\left[h\left(e^{*}\right)=b^{*}\right]\left|S_{\text {real }}\right|=L=1\right] \\
& \geq \operatorname{Pr}_{e^{*} \in U_{E}} \mathbb{E}_{S_{\text {real }} \subseteq E}\left[\operatorname{Pr}_{h \sim \mathcal{H}\left(\mathcal{D}_{S_{\text {ideal }}}\right)}\left[h\left(e^{*}\right)=b^{*}\right]\left|S_{\text {real }}\right|=L-1\right]-O\left(\frac{t(n)}{L}\right) \\
& \geq \frac{1}{2}+\frac{1}{n^{c}}-O\left(\frac{t(n)}{L}\right) \geq \frac{1}{2}-O\left(\frac{1}{n^{c}}\right) .
\end{aligned}
$$

In the equations above, recall that $S_{\text {ideal }}=S_{\text {real }} \cup\left\{\left(e^{*}, b^{*}\right)\right\}$, the first inequality used Equation (17), the second inequality used the same analysis as in Equation (15) and the last inequality used $L=2 n^{c} t(n)$. This shows that $\mathrm{Adv}_{\text {real }}$, on input a uniformly random $e^{*}=\mathrm{Enc}_{\mathrm{K}_{\mathrm{pub}}, r^{*}}\left(b^{*}\right)$ from the set $\mathcal{E}$, outputs $b^{*}$ with probability at least $1 / 2+O\left(n^{-c}\right)$.

It remains to show that Adv satisfies Equation (12), which is equivalent to

$$
\left|2 \underset{K, r^{*}, b^{*}}{\operatorname{Pr}}\left[\operatorname{Adv}\left(\mathrm{K}_{\mathrm{pub}}, e_{\mathrm{K}_{\mathrm{pub}}, r^{*}}\left(b^{*}\right)\right)=b^{*}\right]-1\right| .
$$

Letting Adv be $A_{d v}$ real in the calculation above, we have

$$
\left|2 \operatorname{Pr}_{K, r^{*}, b^{*}}\left[\operatorname{Adv}\left(\mathrm{K}_{\mathrm{pub}}, e_{\mathrm{K}_{\mathrm{pub}}, r^{*}}\left(b^{*}\right)\right)=b^{*}\right]-1\right| \geq O\left(\frac{1}{n^{c}}\right)=\frac{1}{\operatorname{poly}(n)},
$$

\footnotetext{
${ }^{17}$ Note that we have assumed for simplicity that (i) $d_{\mathrm{K}_{\text {priv }}}\left(e_{\mathrm{K}_{\mathrm{pub}}, r}(b)\right)=b$ with probability 1 and (ii) the quantum learning algorithm outputs a hypothesis with probability 1 . If we take into account the decryption probability error $\varepsilon(n)$ and that the learning algorithm succeeded with probability $1-\delta$, then the lower bound in Equation (15) would be $\frac{1}{2}+(1-\delta) n^{-c}-\varepsilon(n)$ and the remaining part of the analysis remains the same.
} 
where the inequality used Equation (19). This concludes the proof of the theorem.

\subsection{Hardness of learning $\mathrm{TC}_{2}^{0}$}

We show here a conditional hardness of quantum learning depth-2 threshold circuits.

Theorem 5.2 Let $n \in \mathbb{N}$. Assuming that the $n$-dimensional LWE problem is hard for a poly $(n)$-time quantum computer, there is no poly $(n)$-time weak quantum-PAC learner for the concept class of $\mathrm{TC}_{2}^{0}$ circuits on $\widetilde{O}(n)$-bit inputs.

The main point of difference between the proof of Theorem 5.2 and the (classical) result of Klivans and Sherstov [KS09] is in the connection between quantum learning and public-key quantum cryptosystems which we already discussed in the previous section. The remaining part of our proof follows their structure very closely. For brevity, we state a simple lemma from their paper without a proof.

Lemma 5.3 [KS09, Lemma 4.1] Fix $\varepsilon>0$ to be a constant. Assume that the intersection of $n^{\varepsilon}$ light half-spaces is weakly quantum-PAC-learnable. Then for every constant $c>0$, the intersection of $n^{c}$ light degree-2 PTFs are weakly quantum-PAC learnable. ${ }^{18}$

We are now ready to prove our main theorem.

Proof of Theorem 5.2. In order to prove the hardness of $\mathrm{TC}_{2}^{0}$, we first consider a subclass of $\mathrm{TC}_{2}^{0}$, intersection of polynomially-many half-spaces, and prove the conditional quantum hardness of learning this subclass.

Fix $\varepsilon>0$ to be a constant. Let $\mathcal{C}$ be the concept class of $n^{\varepsilon}$ light half-spaces and $\mathcal{C}^{\prime}$ be the concept class of degree-2 light PTFs. Let us assume that $\mathcal{C}$ is quantum-PAC learnable. Then by Lemma 5.3, the assumed learnability of $\mathcal{C}$ implies the quantum-PAC learnability of $\mathcal{C}^{\prime}$. By Lemma 3.7, the decryption function of the LWE-cryptosystem is in $\mathcal{C}^{\prime}$. Using Theorem 5.1, we now relate the quantum-PAC learnability of $\mathcal{C}^{\prime}$ to the LWE-cryptosystem as follows: suppose that there exists a quantum-PAC learning algorithm for $\mathcal{C}^{\prime}$, then Theorem 5.1 implies the existence of a distinguisher that can differentiate the encryptions of 0 and 1 in the LWE-cryptosystem. As a consequence, by Theorem 3.6 this would result in a quantum polynomial-time distinguisher for LWE. Using Claim 2.5, we have that $\mathcal{C} \subseteq \mathrm{TC}_{2}^{0}$. Putting this together with our observation in the previous paragraph about quantum PAC learnability of $\mathcal{C}$, we get the theorem statement.

\section{References}

[AAD $\left.{ }^{+} 15\right]$ J. Adcock, E. Allen, M. Day, S. Frick, J. Hinchliff, M. Johnson, S. Morley-Short, S. Pallister, A. Price, and S. Stanisic. Advances in quantum machine learning, 9 Dec 2015. arXiv:1512.02900. 18

[Aar05] Scott Aaronson. Ten semi-grand challenges for quantum computing theory. https: / / www. scottaaronson.com/writings/qchallenge.html, 2005. , 2, 6, 10

[ABHS19] Amir Abboud, Karl Bringmann, Danny Hermelin, and Dvir Shabtay. Seth-based lower bounds for subset sum and bicriteria path. In Proceedings of the Thirtieth Annual ACMSIAM Symposium on Discrete Algorithms, SODA, pages 41-57, 2019. arXiv:1704.04546. 13

\footnotetext{
${ }^{18}$ In [KS09, Lemma 4.1], they state the classical version of this lemma. The exact same proof goes through when we replace classical learners by quantum learners.
} 
$\left[\mathrm{ACL}^{+} 19\right]$ Srinivasan Arunachalam, Sourav Chakraborty, Troy Lee, Manaswi Paraashar, and Ronald de Wolf. Two new results about quantum exact learning. In 46th International Colloquium on Automata, Languages, and Programming, ICALP, pages 16:1-16:15, 2019. arXiv:1810.00481. 5

[AG11] Sanjeev Arora and Rong Ge. New algorithms for learning in presence of errors. In International Colloquium on Automata, Languages, and Programming, pages 403-415. Springer, 2011. 32

[AMRR11] Andris Ambainis, Loïck Magnin, Martin Roetteler, and Jérémie Roland. Symmetryassisted adversaries for quantum state generation. In Proceedings of the 26th Annual IEEE Conference on Computational Complexity, CCC, pages 167-177, 2011. arXiv:1012.2112. 6, 8, 35

[Ang87] D. Angluin. Queries and concept learning. Machine Learning, 2(4):319-342, 1987. 17

[AS05] Alp Atic1 and Rocco Servedio. Improved bounds on quantum learning algorithms. Quantum Information Processing, 4(5):355-386, 2005. 5

[AS09] Alp Atıc1 and Rocco Servedio. Quantum algorithms for learning and testing juntas. Quantum Information Processing, 6(5):323-348, 2009. arXiv:0707.3479. 5, 42

[AS18] Divesh Aggarwal and Noah Stephens-Davidowitz. (gap/s)eth hardness of SVP. In Proceedings of the 50th Annual ACM SIGACT Symposium on Theory of Computing, STOC, pages 228-238, 2018. arXiv:1712.00942. 13

[AW17] Srinivasan Arunachalam and Ronald de Wolf. Guest column: A survey of quantum learning theory. SIGACT News, 48(2):41-67, 2017. arXiv:1701.06806. 17, 18

[AW18] Srinivasan Arunachalam and Ronald de Wolf. Optimal quantum sample complexity of learning algorithms. Journal of Machine Learning Research, 19, 2018. Earlier version in CCC'17. arXiv:1607.00932. 5

[BBS86] Lenore Blum, Manuel Blum, and Mike Shub. A simple unpredictable pseudo-random number generator. SIAM Journal on Computing, 15(2):364-383, 1986. 7, 11

$\left[\mathrm{BDF}^{+} 11\right]$ Dan Boneh, Özgür Dagdelen, Marc Fischlin, Anja Lehmann, Christian Schaffner, and Mark Zhandry. Random oracles in a quantum world. In Advances in Cryptology - ASIACRYPT 2011 - 17th International Conference on the Theory and Application of Cryptology and Information Security, Seoul, South Korea, December 4-8, 2011. Proceedings, pages 41-69, 2011. 16

[BGK18] Sergey Bravyi, David Gosset, and Robert Koenig. Quantum advantage with shallow circuits. Science, 362(6412):308-311, 2018. 5

[BJ99] Nader H. Bshouty and Jeffrey C. Jackson. Learning DNF over the uniform distribution using a quantum example oracle. SIAM Journal on Computing, 28(3):11361153, 1999. Earlier version in COLT'95. 2, 4, 5, 12, 16

[BKST19] Adam Bene Watts, Robin Kothari, Luke Schaeffer, and Avishay Tal. Exponential separation between shallow quantum circuits and unbounded fan-in shallow classical circuits. In Proceedings of the 51st Annual ACM SIGACT Symposium on Theory of Computing, STOC, pages 515-526, 2019. arXiv:1906.08890. 5 
[BKW03] Avrim Blum, Adam Kalai, and Hal Wasserman. Noise-tolerant learning, the parity problem, and the statistical query model. Journal of the ACM (JACM), 50(4):506-519, 2003. 32

$\left[\mathrm{BLP}^{+} 13\right]$ Zvika Brakerski, Adeline Langlois, Chris Peikert, Oded Regev, and Damien Stehlé. Classical hardness of learning with errors. In Symposium on Theory of Computing Conference, STOC'13, Palo Alto, CA, USA, June 1-4, 2013, pages 575-584, 2013. 21

[BPR12] Abhishek Banerjee, Chris Peikert, and Alon Rosen. Pseudorandom functions and lattices. In Advances in Cryptology - EUROCRYPT 2012 - 31st Annual International Conference on the Theory and Applications of Cryptographic Techniques., pages 719-737, 2012. $10,23,24,25,26$

[BV97] Ethan Bernstein and Umesh V. Vazirani. Quantum complexity theory. SIAM Journal on Computing, 26(5):1411-1473, 1997. Earlier version in STOC'93. 2, 4

[CL18] Kai-Min Chung and Han-Hsuan Lin. On the sample complexity of PAC learning quantum process, 2018. arXiv:1810.10938. 12

[CN11] Yuanmi Chen and Phong Q. Nguyen. BKZ 2.0: Better Lattice Security Estimates. In Advances in Cryptology - ASIACRYPT 2011 - 17th International Conference on the Theory and Application of Cryptology and Information Security, Seoul, South Korea, December 48, 2011. Proceedings, pages 1-20, 2011. 3, 11, 32

[DS16] Amit Daniely and Shai Shalev-Shwartz. Complexity theoretic limitations on learning DNF's. In Proceedings of the 29th Conference on Learning Theory (COLT'16), 2016. 13

[FK09] Lance Fortnow and Adam R. Klivans. Efficient learning algorithms yield circuit lower bounds. Journal Computer System Science, 75(1):27-36, 2009. Earlier in COLT'06. 10

[FSA99] Yoav Freund, Robert Schapire, and Naoki Abe. A short introduction to boosting. Journal-Japanese Society For Artificial Intelligence, 14:771-780, 1999. 16

[GG00] Oded Goldreich and Shafi Goldwasser. On the limits of nonapproximability of lattice problems. Journal of Computer and System Sciences, 60(3):540-563, 2000. 21

[GHR92] Mikael Goldmann, Johan Håstad, and Alexander A. Razborov. Majority gates VS. general weighted threshold gates. Computational Complexity, 2:277-300, 1992. 3, 6

[GKZ19] Alex Bredariol Grilo, Iordanis Kerenidis, and Timo Zijlstra. Learning with Errors is easy with quantum samples. Phys. Rev. A, 99:032314, 2019. 5, 21

[HAB02] William Hesse, Eric Allender, and David A Mix Barrington. Uniform constant-depth threshold circuits for division and iterated multiplication. Journal of Computer and System Sciences, 65(4):695-716, 2002. 10, 26, 27

[HV06] Alexander Healy and Emanuele Viola. Constant-depth circuits for arithmetic in finite fields of characteristic two. In Proceedings of the 23rd Annual Conference on Theoretical Aspects of Computer Science, STACS’06, pages 672-683, Berlin, Heidelberg, 2006. Springer-Verlag. 27

[Jac97] Jeffrey C. Jackson. An efficient membership-query algorithm for learning DNF with respect to the uniform distribution. Journal of Computer and System Sciences, 55(3):414-440, 1997. Earlier version in FOCS'94. 4 
[Kha92] Michael Kharitonov. Cryptographic lower bounds for learnability of boolean functions on the uniform distribution. In Proceedings of the Fifth Annual ACM Conference on Computational Learning Theory, COLT, pages 29-36, 1992. 6, 28

[Kha93] Michael Kharitonov. Cryptographic hardness of distribution-specific learning. In Proceedings of the Twenty-Fifth Annual ACM Symposium on Theory of Computing, pages 372-381, 1993. 5, 6, 7, 11, 12, 27, 28, 33

[KLGR16] Iordanis Kerenidis, Mathieu Laurière, Francois le Gall, and Mathys Rennela. Information cost of quantum communication protocols. Quantum Information $\mathcal{E}$ Computation, 16(3\&4):181-196, 2016. 15

[KRS18] Varun Kanade, Andrea Rocchetto, and Simone Severini. Learning DNFs under product distributions via $\mu$-biased quantum fourier sampling. arXiv preprint arXiv:1802.05690, 2018. 4

[KS09] Adam R. Klivans and Alexander A. Sherstov. Cryptographic hardness for learning intersections of halfspaces. Journal of Computer and System Sciences, 75(1):1-12, 2009. Earlier version in FOCS'06. 6, 8, 9, 12, 22, 34, 37

[KV94] Michael J. Kearns and Leslie G. Valiant. Cryptographic limitations on learning boolean formulae and finite automata. Journal of the ACM, 41(1):67-95, 1994. 6, 8, 34

[LLL82] Arjen K. Lenstra, Hendrik W. Lenstra, and László Lovasz. Factoring polynomials with rational coefficients. Mathematische Annalen, 261(4):515-534, Dec 1982. 3, 11, 32

[LMN93] Nati Linial, Yishay Mansour, and Noam Nisan. Constant depth circuits, fourier transform, and learnability. Journal of the ACM, 40(3):607-620, 1993. Earlier in FOCS'89. , $5,11,12,33$

[LPR13a] Vadim Lyubashevsky, Chris Peikert, and Oded Regev. On ideal lattices and learning with errors over rings. Journal of the ACM, 60(6):43:1-43:35, 2013. Preliminary version in EUROCRYPT 2010. 3, 22, 26

[LPR13b] Vadim Lyubashevsky, Chris Peikert, and Oded Regev. A toolkit for ring-lwe cryptography. In Thomas Johansson and Phong Q. Nguyen, editors, Advances in Cryptology - EUROCRYPT 2013, pages 35-54, Berlin, Heidelberg, 2013. Springer Berlin Heidelberg. $10,22,26,27$

[LR19] Nathan Lindzey and Ansis Rosmanis. A tight lower bound for non-coherent index erasure, 2019. arXiv:1902.07336. 6, 8, 35

[LS15] Adeline Langlois and Damien Stehlé. Worst-case to average-case reductions for module lattices. Designs, Codes and Cryptography, 75(3):565-599, Jun 2015. 23

[Mah18] Urmila Mahadev. Classical verification of quantum computations. In Proceedings of 59th Annual IEEE Symposium on Foundations of Computer Science (FOCS), pages 259267, 2018. arXiv:1804.01082. 5

[MSS91] Wolfgang Maass, Georg Schnitger, and Eduardo D. Sontag. On the computational power of Sigmoid versus Boolean threshold circuits. In 32nd Annual Symposium on Foundations of Computer Science, pages 767-776, 1991. 3, 6, 10, 19 
[MT98] Alexis Maciel and Denis Thérien. Threshold circuits of small majority-depth. Information and computation, 146(1):55-83, 1998. 27

[NR04] Moni Naor and Omer Reingold. Number-theoretic constructions of efficient pseudorandom functions. Journal of the ACM, 51(2):231-262, 2004. Earlier version in FOCS'97. 12

[OS17] Igor Carboni Oliveira and Rahul Santhanam. Conspiracies between learning algorithms, circuit lower bounds, and pseudorandomness. In 32nd Computational Complexity Conference, CCC, pages 18:1-18:49, 2017. arXiv:1611.01190. 13

[Pei09] Chris Peikert. Public-key cryptosystems from the worst-case shortest vector problem: extended abstract. In Proceedings of the 41st Annual ACM Symposium on Theory of Computing, STOC, pages 333-342, 2009. 21

[Pei16] Chris Peikert. A decade of lattice cryptography. Foundations and Trends in Theoretical Computer Science, 10(4):283-424, 2016. 21, 23

[PRS17] Chris Peikert, Oded Regev, and Noah Stephens-Davidowitz. Pseudorandomness of ring-lwe for any ring and modulus. In Proceedings of the 49th Annual ACM SIGACT Symposium on Theory of Computing, STOC 2017, pages 461-473, New York, NY, USA, 2017. ACM. 23

[Reg09] Oded Regev. On lattices, learning with errors, random linear codes, and cryptography. Journal of the ACM, 56(6):34:1-34:40, 2009. Earlier version in STOC'05. 12, 21

[RT92] John H Reif and Stephen R. Tate. On threshold circuits and polynomial computation. SIAM Journal on Computing, 21(5):896-908, 1992. 26

[SG04] Rocco Servedio and Steven Gortler. Equivalences and separations between quantum and classical learnability. SIAM Journal on Computing, 33(5):1067-1092, 2004. Combines earlier papers from ICALP'01 and CCC'01. quant-ph/0007036. 5

[Sho97] Peter W. Shor. Polynomial-time algorithms for prime factorization and discrete logarithms on a quantum computer. SIAM Journal on Computing, 26(5):1484-1509, 1997. Earlier version in FOCS'94. quant-ph/9508027. 5, 6

[SP18] Maria Schuld and Francesco Petruccione. Supervised Learning with Quantum Computers. Springer, 2018. 18

[Sta19] Katherine E Stange. Algebraic aspects of solving ring-lwe, including ring-based improvements in the blum-kalai-wasserman algorithm. arXiv preprint arXiv:1902.07140, 2019. 3

[Tou15] Dave Touchette. Quantum information complexity. In Proceedings of the Forty-Seventh Annual ACM on Symposium on Theory of Computing, STOC 2015, pages 317-326, 2015. arXiv:1404.3733. 15

[Val84] Leslie Valiant. A theory of the learnable. Communications of the ACM, 27(11):11341142, 1984. 1, 3, 6, 16

[Ver90] Karsten A. Verbeurgt. Learning DNF under the uniform distribution in quasipolynomial time. In Proceedings of the 3rd Annual Workshop on Computational Learning Theory (COLT'90), pages 314-326, 1990. 2 
[Wag02] David Wagner. A generalized birthday problem. In Annual International Cryptology Conference, pages 288-304. Springer, 2002. 32

[Zha12] Mark Zhandry. How to construct quantum random functions. In 53rd Annual IEEE Symposium on Foundations of Computer Science, FOCS 2012, pages 679-687, 2012. 10, 25,26

\section{A Time-efficient learning of $\mathrm{NC}^{0}$}

We prove the following theorem, which is straightforward from known results in quantum learning theory. Since we didn't find an explicit reference to the proof of such an explicit theorem in literature, we make it formal here.

Theorem A.1 Let $c>0$ be a constant. Let $\mathcal{C}$ be the concept class of all Boolean functions on $n$ bits which are computable by polynomial number of gates with at most 2 inputs and depth at most $d$. Then $\mathcal{C}$ can be learned under the uniform distribution with error at most $\varepsilon$, using at most $\widetilde{O}\left(2^{d} / \varepsilon\right)$ quantum examples, $O\left(2^{2^{d}}\right)$ classical examples and time $\widetilde{O}\left(n 2^{d} / \varepsilon+2^{2^{d}} \log (1 / \varepsilon)\right)$.

The proof directly follows from Atıcı and Servedio's theorem on learning $k$-juntas.

Theorem A.2 ([AS09]) There exists a quantum algorithm for learning $k$-juntas under the uniform distribution that uses $O((k \log k) / \varepsilon)$ uniform quantum examples, $O\left(2^{k}\right)$ uniform classical examples, and $\widetilde{O}\left(n k / \varepsilon+2^{k} \log (1 / \varepsilon)\right)$ time.

Now we proceed with proving our theorem.

Proof of Theorem A.1. Suppose that $f:\{0,1\}^{n} \rightarrow\{0,1\}$ is computed by a circuit with depth at most $d$. Since the gates of the circuit have fan-in at most 2, clearly the output of the circuit for $f$ (on an arbitrary input $x$ ) depends only on $2^{d}$ bits of $x$. Hence every $f \in \mathcal{C}$ is a $2^{d}$-junta. Using Theorem A.2, it follows that the concept class of $2^{d}$-juntas can be learned using $\widetilde{O}\left(2^{d} / \varepsilon\right)$ quantum examples, $O\left(2^{2^{d}}\right)$ classical examples and time $\widetilde{O}\left(n 2^{d} / \varepsilon+2^{2^{d}} \log (1 / \varepsilon)\right)$.

Corollary A.3 Let $c>0$ be a constant. Let $\mathcal{C}$ be the concept class of $n$-bit functions which are computable by polynomial number of gates with fan-in at most 2 , depth at most $\log (c \log n)$. Then $\mathcal{C}$ can be learned using at most $\widetilde{O}(n / \varepsilon)$ quantum examples, $O\left(n^{c}\right)$ classical examples and time $\widetilde{O}\left(n^{c} / \varepsilon\right)$.

Proof. The proof immediately follows from Theorem A.1 by plugging in $d=\log (c \log n)$.

Observe that, since $\mathrm{NC}^{0}$ is the class of circuits on $n$-bits with depth $O(1), \mathrm{NC}^{0}$ is contained in the concept class $\mathcal{C}$ considered in the corollary above. 